This document is confidential and is proprietary to the American Chemical Society and its authors. Do not copy or disclose without written permission. If you have received this item in error, notify the sender and delete all copies.

\title{
Phase Change Ultrasound Contrast Agents with a Photopolymerized Diacetylene Shell
}

\begin{tabular}{|c|c|}
\hline Journal: & Langmuir \\
\hline Manuscript ID & la-2019-01160a \\
\hline Manuscript Type: & Article \\
\hline $\begin{array}{r}\text { Date Submitted by the } \\
\text { Author: }\end{array}$ & 19-Apr-2019 \\
\hline Complete List of Authors: & $\begin{array}{l}\text { Toumia, Yosra; Universita degli Studi di Roma Tor Vergata, Dipartimento } \\
\text { di Scienze e Tecnologie Chimiche } \\
\text { Cerroni, Barbara; Universita degli Studi di Roma Tor Vergata, } \\
\text { Dipartimento di Scienze e Tecnologie Chimiche } \\
\text { Domenici, Fabio; Universita degli Studi di Roma Tor Vergata, } \\
\text { Dipartimento di Scienze e Tecnologie Chimiche } \\
\text { Lange, Heiko; Universita degli Studi di Roma Tor Vergata, Dipartimento } \\
\text { di Scienze e Tecnologie Chimiche } \\
\text { Bianchi, Livia; Universita degli Studi di Roma Tor Vergata, Dipartimento } \\
\text { di Scienze e Tecnologie Chimiche } \\
\text { Cociorb, Madalina; Universita degli Studi di Roma Tor Vergata, } \\
\text { Dipartimento di Scienze Tecnologie Chimiche } \\
\text { Brasili, Francesco; Universita degli Studi di Roma Tor Vergata, } \\
\text { Dipartimento di Scienze eTecnologie Chimiche } \\
\text { Chiessi, Ester; Universita degli Studi di Roma Tor Vergata, Dipartimento } \\
\text { di Scienze e Tecnologie Chimiche } \\
\text { D'Agostino, Emiliano; DoseVue NV, Belgium } \\
\text { Van Den Abeele, Koen; KU Leuven, Department of Physics } \\
\text { Heymans, Sophie; KU Leuven, Department of Physics } \\
\text { D'Hooge, Jan; Medical Center } \\
\text { Paradossi, Gaio; Universita degli Studi di Roma Tor Vergata, } \\
\text { Dipartimento di Scienze e Tecnologie Chimiche }\end{array}$ \\
\hline
\end{tabular}

\section{SCHOLARONE ${ }^{\text {tw }}$ Manuscripts}




\section{Phase Change Ultrasound Contrast Agents with a}

\section{Photopolymerized Diacetylene Shell}

Yosra Toumia ${ }^{1}$, Barbara Cerroni ${ }^{1}$, Fabio Domenici ${ }^{1}$, Heiko Lange ${ }^{1}$, Livia Bianchi ${ }^{1}$, Madalina Cociorb ${ }^{1}$, Francesco Brasili ${ }^{1}$, Ester Chiessi ${ }^{1}$, Emiliano D'Agostino ${ }^{2}$, Koen Van Den Abeele ${ }^{3}$, Sophie V. Heymans ${ }^{3}$, Jan D’Hooge ${ }^{4}$ and Gaio Paradossi ${ }^{1 *}$

${ }^{1}$ Department of Chemical Sciences and Technologies, University of Rome Tor Vergata, Via della Ricerca Scientifica 1, 00133, Rome, Italy; ${ }^{2}$ DoseVue NV, Philips Open Manufacturing Campus, Slachthuisstraat 96, B-2300 Turnhout, Belgium; ${ }^{3}$ Department of Physics, KU Leuven, KULAK, Kortrijk, Belgium; ${ }^{4}$ Medical Center, KU Leuven, Leuven, Belgium

*Corresponding Author: Gaio Paradossi, paradossi@stc.uniroma2.it

\section{ABSTRACT}

Phase change contrast agents for ultrasound (US) imaging consist of nanodroplets (NDs) with a perfluorocarbon (PFC) liquid core stabilized with a lipid or a polymer shell. Liquid $\leftrightarrow$ gas transition, occurring in the core, can be triggered by US to produce acoustically active microbubbles (MBs) in a process named acoustic droplet vaporization ( $A D V)$. MB shells containing polymerized diacetylene moiety were considered as a good trade off between the lipid MBs, showing optimal attenuation and the polymeric ones displaying an enhanced stability. This work reports on novel perfluoropentane and perfluorobutane NDs stabilized with a monolayer of an amphiphilic fatty acid, i.e. 10,12-pentacosadiynoic acid (PCDA), cured with ultraviolet (UV) irradiation. The photopolymerization of the acetylene groups, evidenced by the appearance of a blue color due to the conjugation of ene-yne sequences, exhibits a chromatic transition from the non-fluorescent blue color to a fluorescent red color when the NDs are heated or the $\mathrm{pH}$ of the suspension is basic. An estimate of the molecular weights reached by the polymerized PCDA in the shell, poly(PCDA), has been obtained using gel permeation chromatography and MALDI-TOF mass spectrometry. The poly(PCDA)/PFC NDs show good biocompatibility with fibroblast cells. ADV efficiency and acoustic properties before and after the transition were tested using a $1 \mathrm{MHz}$ probe, revealing a resonance frequency between $1-2 \mathrm{MHz}$ similar to other lipidic MBs. The surface of PCDA shelled NDs can be easily modified without influencing the stability and the acoustic performances of droplets. As a 
proof of concept we report on the conjugation of cyclic RGD and PEG chains of the particles to support targeting ability toward endothelial cells.

\section{INTRODUCTION}

Microbubbles (MBs) are micron-sized particles encapsulating a gas core and can be regarded as a colloidal system with unique mechanical properties. MBs are able to resonate in the ultrasound frequency range, ${ }^{1,2}$ making them a powerful injectable contrast agent for ultrasound imaging. ${ }^{3,4}$ Recently, MBs have been considered a potential multifunctional platform supporting therapeutics and diagnostics with the capability to target specifically pathological tissues and cells. ${ }^{5}$ The structural element characterizing the overall behavior and properties of MBs is the shell confining the gas core from the dispersing medium which can be made of proteins, lipids or polymers. $^{6}$ The shell of a lipid MB is a spherical surfactant monolayer, with a thickness of few nanometers, stabilized by weak interactions. Differently from lipid MBs, a layer of crosslinked polymer chains with varying extent of water permeation results in MBs having a shell thickness of some hundreds of nanometers. ${ }^{7-9}$ Surprisingly, in some cases the difference in shell thickness does not cause a huge change of the acoustic properties of the two types of MBs. ${ }^{10}$ Lipid MBs are much easier to drive up to inertial cavitation by ultrasound (US) irradiation and have much shorter life-time than polymer shelled MBs, reflected also in a dramatic shortening of the circulation life in a hostile environment for exogenous particles such as blood. ${ }^{11-16}$ Moreover, lipid shelled MBs are less prone for conjugating moieties enabling the active targeting or drug delivery because of the delicate packing at the basis of the surfactant shell layer. ${ }^{17}$ Recently, phase change nanodroplets (NDs) stabilized by lipid, polymeric or surfactant-based shells have been regarded as another way to obtain MBs by in situ US activation. This process, known as acoustic droplet vaporization (ADV), implies the transformation of NDs to MBs as final product and entails interesting features, which can be exploited in biomedicine: ${ }^{18-21}$ (i) in the perfluorocarbon (PFC) core, hydrophobic drugs can be dispersed or solubilized; ${ }^{22}$ (ii) phase change systems, subjected to ADV, have been also considered for the delivery of drugs previously loaded in the liquid core of the starting NDs; ${ }^{23}$ (iii) 
the nanometric size of systemically administered droplets, is a remarkable feature in the presence of enhanced permeability and retention (EPR) effect, typically affecting tumor tissues, and can play a role in the penetration of biological barriers due to the vasculature leakiness. ${ }^{24-28}$ Once the biological barrier is overcome after extravasation, the NDs engineered for active tumor targeting, can be converted into MBs by a US-triggered phase transition. ${ }^{29}$ At this point MBs can be used both to support an enhanced ultrasound imaging and as therapeutic drug carrier and delivery device, bringing MBs to inertial cavitation and causing the local release of drug to the pathological tissue. From the literature it emerges that the energy threshold needed to trigger ADV is linked in a complex manner to the composition and structure of the NDs shell, the elasticity and surface tension of the shell. Poly(lactic-glycolic) acid, PLGA, shelled NDs encapsulating perfluoropentane were used for carrying doxorubicin in the liquid core. A power of $8 \mathrm{~W}$ for 3 min was necessary for vaporizing the core of these NDs, a threshold much higher than the 3 $W$ for few seconds used when the shell is lipidic. ${ }^{30}$ Among lipid shelled NDs, we report here on a particular type which combines the features of the lipid ones with the robustness of a crosslinked structure. Park et al. reported on the incorporation of photopolymerizable diacetylenes into the formulation of phospholipidic MBs as a mean to accomplish tunable shell polymerization and enhanced stability of the microbubbles. ${ }^{31}$ With this scenario in mind, we designed novel submicron-size, phase-change NDs to obtain MBs exhibiting features of both the lipid and the polymer MBs together. The shell encapsulating low boiling point PFCs, i.e. perfluoropentane (PFP) and perfluorobutane (PFB) with boiling points of 29 and -2 ${ }^{\circ} \mathrm{C}$, respectively, is formed by a fatty acid monolayer consisting in 10,12 pentacosadiynoic acid (PCDA). A PCDA molecule exhibits interesting surfactant features with a carboxyl group as polar head and an aliphatic tail bearing two alkyne groups in positions 10 and 12, respectively. The diacetylene moiety during UV curing is subjected to an intermolecular topo-tactic free radical photo-polymerization, yielding an alternate sequence of double and triple conjugated bonds transverse to the PCDA tails and connecting several single PCDA molecules, i.e. a poly(PCDA); the conjugation is evidenced by the appearance of a blue color. The result of the photopolymerization is a nanodroplet encapsulating a liquid PFC with a shell being 
intermediate between a lipid and a polymeric one (see Scheme 1), and exhibiting interesting optical properties already reported in the literature for UV cured PCDA films. $^{32-34}$
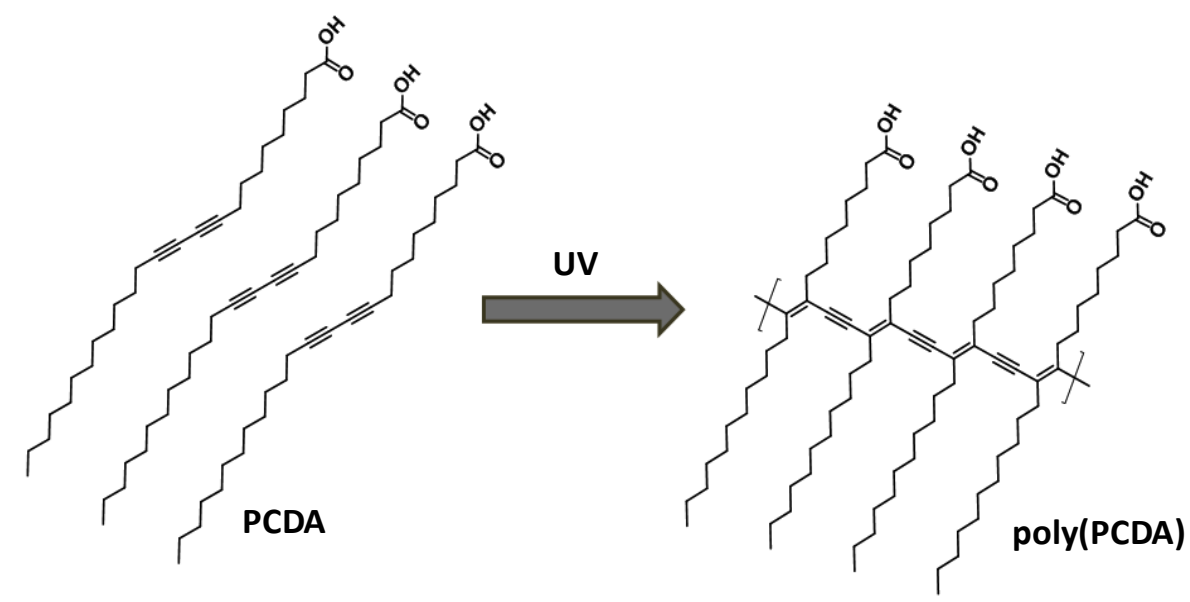

Scheme 1. 1-4 addition polymerization of PCDA during UV curing

Our investigation concerns the stability and the structure of the PCDA shelled nanodroplets after UV curing, the responsiveness to US irradiation and ADV efficiency. We used poly(PCDA) shelled NDs with unusual stability and chemical versatility of the surface, for lipid NDs, as proof of concept to show new specific cells recognition ability and labelling of fluorescence probes. In this respect, the presence of functional diacetylenic and carboxylic moieties on one single PCDA molecule is functional to obtain a great variety of engineered phase shift hybrid devices incorporating near infrared (NIR) absorbing nanomaterials. ${ }^{35-40}$ 


\section{EXPERIMENTAL SECTION}

\section{Materials:}

The following materials were purchased from Sigma Aldrich (Milan, Italy), and used without further purification: 10,12-pentacosadiynoic acid (PCDA), poly(ethylene glycol) 2-aminoethyl ether acetic acid (3000) (amino-PEG-COOH), pluronic F127, fluorescinamine isomer I, 1-Ethyl-3-(3-dimethylaminopropyl) carbodiimide hydrochloride (EDC), N-hydroxy-succinimide (NHS), 2-morpholinoethane sulfonic acid buffer (MES), acetic acid, 1,8,9-anthracenetriol (Dithranol), sodium chloride $(\mathrm{NaCl})$, thiazolyl blue tetrazolium bromide (MTT), Dulbecco's modified Eagle's medium (DMEM), fetal bovine serum (FBS), L-glutamine, penicillin/streptomycin, and human serum. Perfluoropentane (PFP) and perfluobutane (PFB) were purchased from Apollo Scientific, UK; Irgacure 2959 and chloroform were purchased from BASF (Milan, Italy) and Alfa Aesar, respectively. Cyclo(Arg-Gly-Asp-D-Phe-Lys) (cRGD) was purchased from Avanti Polar (Milan, Italy). Methanol (MeOH) and dimethyl sulfoxide (DMSO) were purchased from Carlo Erba, Milan, Italy. Milli-Q (mQ) quality water (18.2 M $\Omega . c m)$ was produced by a Pure Lab deionization apparatus from USF (Perugia, Italy). Murine fibroblast cell line, NIH 3T3, was purchased from Istituto Zooprofilattico della Lombardia e dell'Emilia Romagna (Milan, Italy).

\section{Methods:}

\subsection{Poly(PCDA)/PFC NDs formulation:}

First, a $1 \mathrm{mM}$ aqueous suspension of PCDA monomeric vesicles is prepared (see Supporting Information). Then PCDA/PFP droplets were prepared by adding $50 \mu \mathrm{L}$ of liquid PFP to $1 \mathrm{~mL}$ of PCDA monomer aqueous suspension cooled at $4^{\circ} \mathrm{C}$. The mixture is sonicated using a sonication bath cleaner (Ceia CP104, Florence, Italy) at full power for about $10 \mathrm{~min}$ (two iterations of $5 \mathrm{~min}$ each), until all PFP is incorporated into PCDA, leading to a milky solution. Then $10 \mu \mathrm{L}$ of Pluronic F127 aqueous solution (10 $\mathrm{mg} / \mathrm{mL}$ ) is added as a stabilizer prior to polymerization. The droplets are immersed in an ice bath and photopolymerized under $365 \mathrm{~nm}$ UV light for 15 min using a UV lamp $\left(7 \mathrm{~W} / \mathrm{cm}^{2}\right)$ in the presence of Irgacure 2959 photoiniator $(0.15 \% \mathrm{w} / \mathrm{v})$. The development of a blue color in the suspension is taken as the visual evidence that 
the crosslinking reaction occurred. The UV cured droplets are washed with $\mathrm{mQ}$ water by centrifugation at $5200 \mathrm{rpm}$ (2358 g-force) for $4 \mathrm{~min}$.

As for PCDA/PFB droplets, first an empty vial sealed with a rubber septum is immersed in liquid nitrogen, then PFB gas is fluxed for few seconds inside the vial. As soon as the gas is liquefied, the vial is removed from the liquid nitrogen. Quickly $1 \mathrm{~mL}$ of PCDA aqueous suspension ( $1 \mathrm{mM})$ is added with a syringe and the mixture is immediately placed in a sonication bath for $10 \mathrm{~min}$ at RT until obtaining a milky suspension. A fraction of PFB in the liquid state is incorporated by the PCDA molecules, while the remaining PFB evaporates in the empty volume of the vial, creating a pressure head over the liquid suspension. The droplets are subsequently UV cured for 15 min under $365 \mathrm{~nm}$ light, in presence of Pluronic F127 and Irgacure 2959 at concentrations of $0.02 \%$ and $0.15 \%(w / v)$, respectively.

Both poly(PCDA)/PFP and poly(PCDA)/PFB samples were stored at $4^{\circ} \mathrm{C}$ for further analysis and stability study.

\subsection{PCDA molecular shell density determination}

An estimate of the surface density number of surfactant molecules in the shell of poly(PCDA)/PFP NDs is carried out on a sample fraction with dimensions above 800 $\mathrm{nm}$. Typically, $5 \mathrm{~mL}$ of poly(PCDA)/PFP were prepared as described previously and the largest NDs fraction was separated by centrifugation (2000 rpm, 360 g-force, $5 \mathrm{~min})$. This fraction can be easily detectable and countable in a Neubauer chamber by bright field microscopy (40x). After elution in a column packed with Amberlite IR120, (Merck, GE) proton exchange resin, the carboxyl groups of the PCDA shell in the selected fraction are titrated potentiometrically with a glass combined $\mathrm{pH}$ semimicroelectrode, (Crison, SP). Further details of this procedure are provided in the Supporting Information experimental section S2. 


\subsection{Chromatic Transition Study of poly(PCDA) droplets:}

The chromatic transition of poly(PCDA) NDs upon heating is followed with an UV-vis double beam JASCO V-630 (Milan, Italy) UV-vis spectrophotometer with a $1 \mathrm{~cm}$ quartz cell (Hellma, GE) equipped with a Peltier unit (Jasco EHC-716, Milan, Italy), between $25^{\circ} \mathrm{C}$ and $80^{\circ} \mathrm{C}$. The measurements were recorded taking water as reference and subtracting the spectra of PCDA NDs, before UV curing, to remove the scattering.

\subsection{Dynamic light scattering (DLS):}

The size distribution of poly(PCDA)/PFC NDs is determined by DLS. $1 \mathrm{~mL}$ of a 100 fold diluted solution of PFC filled poly(PCDA) NDs is filled into a cylindrical quartz cuvette. The measurements are carried out at $90^{\circ}$ using a photometer equipped with a $\mathrm{BI}$ 200SM goniometer, a BI-9000AT (Brookhaven Instruments Co.) correlation board and a solid state laser source emitting at $532 \mathrm{~nm}$. Analysis of the autocorrelation function, $g_{2}(q, t)$, of the scattered intensity was carried out using the cumulants algorithm provided as part of the standard software package of the instrument.

\subsection{Z-potential:}

The Z potential of "blue" and "red" NDs (see Section 2.2) suspended in mQ water is measured at $25.0 \pm 0.1{ }^{\circ} \mathrm{C}$ with or without Pluronic F127 at a concentration of 0.1 $\mathrm{mg} / \mathrm{mL}$, used in the PCDA polymerization. Phase Analysis Light Scattering (PALS) technique with a Malvern Nano ZetaSizer apparatus (Malvern Instruments Ltd, UK) equipped with palladium electrode dip cell ZEN 1002 (Malvern, UK) has been used for the measurement of the electrophoretic mobility and the related $Z$ potential, provided herein in the Helmholtz-Smoluchowski approximation. ${ }^{41}$

\subsection{Gel Permeation Chromatography (GPC):}

Sample preparation: $12 \mathrm{~mL}$ of freshly generated poly(PCDA)/PFC NDs are freeze dried, and the resulting powder is suspended just before measurement in DMSO containing $0.1 \%(\mathrm{w} / \mathrm{v})$ lithium chloride, yielding a final concentration of $2 \mathrm{mg} / \mathrm{mL}$.

Measurement: GPC analysis of polymerized NDs is performed using a Shimadzu instrument consisting of a controller unit (CBM-20A), a pumping unit (LC 20AT) equipped with a $20 \mu \mathrm{L}$ sample loop, a degasser unit (DGU-20A3), a column oven (CTO-20AC), a diode array detector (SPD-M20A), and a refractive index detector 
(RID-10A); Shimadzu Lab Solution software package (Version 5.42 SP3) allowed for the system control. APLgel $5 \mu \mathrm{m}$ Mini MIX-C column (Agilent, $250 \times 4.6 \mathrm{~mm}$ ) eluted at $70{ }^{\circ} \mathrm{C}$ with DMSO containing $0.1 \%(\mathrm{~m} / \mathrm{v})$ lithium chloride was used, with a runtime of $20 \mathrm{~min}$ at $0.25 \mathrm{~mL} \mathrm{~min}{ }^{-1}$ flow rate.

Molecular weights are calculated from a linear calibration $\left(R^{2}=0.999\right)$ based on poly(styrene sulfonic acid) polymer fractions ranging from 4.3 to $2600 \mathrm{kDa}$ using a dedicated excel-file reported previously. ${ }^{42}$

\section{7. Matrix-Assisted Laser Desorption/Ionization-Time of Flight (MALDI-ToF) Mass} Analysis

Sample preparation: $1 \mathrm{~mL}$ of freshly generated poly(PCDA)/PFC NDs is centrifuged at $3900 \mathrm{rpm}$ (1326 g-force) for $4 \mathrm{~min}$, and the recovered pellet is suspended in aqueous buffer solutions, i.e., acetate buffer $(0.1 \mathrm{M}, \mathrm{pH} 4.5)$ or phosphate buffer $(0.1 \mathrm{M}, \mathrm{pH}$ 8) at a final concentration of $4 \mathrm{mg} / \mathrm{mL}$. The buffer suspensions of NDs are mixed (1:1 $\mathrm{v} / \mathrm{v}$ ) with a matrix solution of dithranol at $2 \mathrm{mg} / \mathrm{mL}$ in $\mathrm{NaCl}$ aqueous solution (10 $\mathrm{mg} / \mathrm{mL}$ ) just before measurement. $2 \mu \mathrm{L}$ of the resulting analysis solution are placed on the MALDI-TOF sample holder and left for drying in the dark.

Measurement: Measurements are performed on a Voyager-DE PRO Biospectrometry Work station MALDI-TOF mass spectrometer by Applied Biosystems (USA), equipped with a nitrogen laser $(337 \mathrm{~nm})$ using the Applied Biosystems operating and analysis software, monitoring a mass range between 200 - $5000 \mathrm{Da}$ in positive linear mode. The delayed extraction technique is used applying delay times of 200 ns. An accelerating voltage of $25.0 \mathrm{kV}$, a laser intensity of around $2000 \mathrm{eV}$, a grid voltage of $95 \%$, and a guide wire voltage rate of $0.2 \%$ were used for generation of optimum spectra. Spectra were collected in positive and negative mode, adjusting laser intensity as necessary. Analyzed spectra are the sum of 50 to 250 shots. The instrument was calibrated using commercially available PEG standards in the range between 500 and 10,000 Da.

Mass analysis and spectra interpretation was achieved using Applied Biosystems Data Explorer and a combinatorial excel-based analysis. 


\section{8. Bright field, transmission and CLSM microscopies:}

Although close to instrumental resolution, an overall view of the different droplet samples is carried out using an inverted Eclipse model Ti-E microscope (Nikon Instruments, Japan) equipped with a $40 \times$ long-working distance objective, a $60 \times / 1.4$ oil immersion Plan Apo objective, and an $\mathrm{Ar}^{+}$green laser $\left(\lambda_{\mathrm{exc}}=488 \mathrm{~nm}\right)$ (Melles Griot, Carlsbad, CA), and a He-Ne laser $\left(\lambda_{\text {exc }}=543 n m\right)$ (Spectra Physics, Mountain View, CA). An estimation of the poly(PCDA)/PFC droplets concentrations is assessed by bright field microscopy using a Nauber chamber $(0.25 \times 0.25 \times 0.1) \mu \mathrm{m}$ and imageJ software (freeware) to analyze micrographs. ADV is monitored by fluorescence and confocal microscopy for fluoresceinamine conjugated PCDA/PFP droplets ( $\lambda$ exc 488 $\mathrm{nm})$.

\section{9. Acoustic droplets vaporization (ADV):}

The ADV experiments are performed using a SP100 sonoporator $(1 \mathrm{MHz})$ from SONIDEL $^{\mathrm{TM}}$ (Dublin, IE). Typically, for each polymerized droplets systems described above, an "Ibidi" channel slide $(50 \times 5 \times 0.4) \mathrm{mm}$ (Milan, Italy) is filled with $200 \mu \mathrm{L}$ of 10 fold diluted droplets suspension and fixed in a polystyrene flask degassed water bath placed on the microscope. An ultrasound probe is placed in the water bath at $37^{\circ} \mathrm{C}$ and at $2 \mathrm{~cm}$ height. Acoustic vaporization is carried out at $1 \mathrm{MHz}$ central frequency, $100 \%$ duty cycle for $30 \mathrm{~s}$ and at different US power to assess the efficiency of each type of PFC filled poly(PCDA) droplets. The transition NDs $\rightarrow$ MBs is followed by bright field microscopy. For these experiments droplets in the channel were diluted in human serum.

\subsection{Attenuation Measurements:}

The experimental setup is described in Scheme S1 in the Supporting Information. Typically, a glass cell $(2 \mathrm{~cm} \times 2 \mathrm{~cm} \times 4 \mathrm{~cm})$ is filled with aqueous droplet suspensions in degassed DMEM (at an estimated concentration of $3 \times 10^{7} \mathrm{NDs} / \mathrm{mL}$ ) The latter features two openings at opposite sides separated by a distance of $2 \mathrm{~cm}$ allowing contact with the emitting and receiving transducers. Two flat $10 \mathrm{MHz}$ transducers (Olympus V311) as emitter and receiver and a waveform generator producing consecutive sinusoidal ultrasound bursts at frequencies ranging from 0.5 to $20 \mathrm{MHz}$ 
(in steps of $0.25 \mathrm{MHz}$ ) are used. A constant input voltage of $6 \mathrm{~V}$ was applied at each frequency, resulting in acoustic pressure levels below $60 \mathrm{kPa}$. The attenuation was deduced from the transmitted signals detected by the receiver by means of a reference method, where the reference is the DMEM medium. Acoustic attenuation measurements of poly(PCDA)/PFP and poly(PCDA)/PFB NDs are performed before and after ADV. For the ADV attenuation measurements, the sonoporator head is carefully immersed on the top of the cell, and the droplets are irradiated with the same parameters described in the ADV section above (Duty cycle 100, 0.1-3.6 $\left.\mathrm{W} / \mathrm{cm}^{2}, 30 \mathrm{~s}\right)$.

\subsection{MTT cytotoxicity evaluation of poly(PCDA)PFP droplets:}

For MTT assays, typically, $5 \times 10^{4}$ of fibroblasts NIH 3 T3 are seeded in 24-well plates and cultured in DMEM supplemented with $2 \mathrm{mM}$ L-glutamine, $1 \%$ penicillin/streptomycin and $10 \% \mathrm{FBS}$ at $37^{\circ} \mathrm{C}$ in a humidified atmosphere containing $5 \% \mathrm{CO}_{2}$. The multi-well plates containing the cells were left for 12 hours in incubator (HeraCell 150i, Thermo-Scientific) to allow cell adhesion. Afterwards, $900 \mu \mathrm{l}$ of DMEM and $100 \mu \mathrm{L}$ corresponding to amounts ranging from about $5 \times 10^{8}$ to $10^{5}$ (droplets $/ \mathrm{mL}$ ) of poly(PCDA)/PFP droplets were added. After $24 \mathrm{~h}$ contact, the cells are washed twice with PBS and incubated for $4 \mathrm{~h}$ with MTT at a final concentration of $0.5 \mathrm{mg} / \mathrm{ml}$ in serum-free DMEM, at $37{ }^{\circ} \mathrm{C}$ under $5 \% \mathrm{CO}_{2}$. Then, MTT is removed and the water-insoluble formazan crystals formed as by-product of the test are dissolved in DMSO. Spectrophotometer absorbance measurements at $570 \mathrm{~nm}$ and $650 \mathrm{~nm}$ wavelengths were carried out. The cell viability is expressed according to eq. 1 as percentage value representing mitochondrial activity. Untreated cells (NT), i.e. without being in contact with the droplets, are used as control.

$$
\text { Cell Viability }(\%)=\frac{(A b s N D S)_{570}-(A b s N D S)_{650}}{(A b s N T)_{570}-(A b S N T)_{650}} \times 100
$$

where Abs is the absorbance and NDs represents a poly(PCDA)/PFP droplets treated cells. Data were statistically analyzed using the paired Student t-test within the groups. $^{43}$ 


\subsection{Functionalization of poly(PCDA)/PFP NDs}

\section{Poly(PCDA)/PFP fluoresceinamine labeled droplets}

Fluoresceinamine dye is conjugated to the poly(PCDA)/PFP (blue) sub-micron droplets by EDC/NHS chemistry through the carboxylic moiety of the PCDA monolayer at the PFP/water interface and the $-\mathrm{NH}_{2}$ group of the fluorescent label (see Scheme S3 in the Supporting Information). Typically, the carboxylic groups of the polymerized droplets, prepared as described in Section 2.1, are activated with an equimolar amount of EDC/NHS (approx. $2 \mu \mathrm{mol}$ ) at $\mathrm{pH}$ fixed to 6.5 with MES buffer $(0.1 \mathrm{M})$ and keeping the suspension under stirring in the dark at RT for $30 \mathrm{~min}$. Then $2 \mu \mathrm{mol}$ of dye solution in $\mathrm{MeOH} / \mathrm{H}_{2} \mathrm{O}(1: 1 \mathrm{v} / \mathrm{v})$ are added and the suspension is kept under stirring for 2 hours. Finally the covalently labeled droplets are washed with $\mathrm{mQ}$ water by a multi-step centrifugation at $5200 \mathrm{rpm}$ (2358 g-force) for $4 \mathrm{~min}$ to remove unbound dye.

RGD-functionalized PCDA/PFC droplets for in vitro cell targeting:

Preparation: The strategy for the tagging of poly(PCDA) NDs with $c R G D$ peptide consists in a double EDC/NHS chemistry. In a first step, a PEG chain bearing $\mathrm{NH}_{2}$ and $\mathrm{COOH}$ as end groups is tethered on the shell of the NDs via EDC/NHS leading to an amide bond through the primary amine group of PEG and the carboxyl groups on the NDs surface. Typically $1 \mu \mathrm{mol}$ of NDs is suspended in a MES buffer $(0.1 \mathrm{M}, \mathrm{pH} 6.5)$, and activated by EDC/NHS $(2 \mu \mathrm{mol})$ for $30 \mathrm{~min}$ following the addition of $2 \mu \mathrm{mol}$ of amino-PEG-COOH. The reaction is carried out for $2 \mathrm{~h}$ at $\mathrm{RT}$ under gentle stirring. Then NDs are washed with MES buffer to eliminate unbound PEG chains by centrifugation (5000 rpm/2180 g-force). In a second step, the resulting droplets are activated by 0.5 $\mu \mathrm{mol}$ EDC/NHS for 30 min following the addition of CRGD bearing a D-Phe-Lys moiety (0.5 $\mu \mathrm{mol})$. Thereafter, the functionalized cRGD NDs (poly(PCDA)-PEG-cRGD/PFP) are washed by centrifugation (5000rpm/2180 g-force, $4 \mathrm{~min}$ ) and heated at $70^{\circ} \mathrm{C}$ to pass from the blue to the red form, with a partial decrease of the average dimensions. This step is necessary in order to attribute fluorescence to the NDs for an easy detection in the adhesion tests, which allows avoiding a further labelling step of NDs. In vitro cell targeting: HUVEC cells were cultured in DMEM supplemented with $2 \mathrm{mM}$ I-glutamine, $10 \% \mathrm{FBS}$ and $1 \%$ penicillin/streptomycin at $37^{\circ} \mathrm{C}$ in a humidified 
atmosphere containing $5 \% \mathrm{CO}_{2}$. In a typical flow experiment, flow chamber slides ( $\mu$ Slides | 0.4 ) are coated with $2 \%$ gelatin solution according to the Ibidi protocol, after which $10^{5}$ of HUVEC cells are seeded. To allow cells to grow and adhere on the bottom of the chamber slides, $\mu$-Slides I 0.4 were put in the incubator (HeraCell 150i, Thermo Fisher Scientific) and left overnight. Afterwards, cells are washed three times with PBS/FBS/DMEM (40\%, 40\%, 10\%) $(\mathrm{v} / \mathrm{v})$ and placed under an inverted microscope (Nikon Inverted Microscope Eclipse Ti-E) equipped with a 40x objective (Nikon, Japan), a motorized stage and the Zylas CMOS camera 4.2 (Andor, Belfast, UK). The setup, assembled as described in Scheme 2, consists of an infusion pump with a $10-\mathrm{mL}$ syringe filled with the NDs $(0.1 \mathrm{mM})$ suspension in the PBS/FBS/DMEM mixture and connected by silicon tubing $(20 \mathrm{~cm}$, internal diameter $1.6 \mathrm{~mm}$ and outer diameter $3.2 \mathrm{~mm}$ ) and Luer-lock connector adapters to the $\mu$-Slide. HUVEC cells were grown at confluence on the bottom side of the channel slide. The poly(PCDA)PEG/PFP and poly(PCDA)PEG-CRGD/PFP NDs, suspended in cell medium containing FBS, were fluxed for $10 \mathrm{~min}$ at a shear stress corresponding to $1 \mathrm{dyn} / \mathrm{cm}^{2}$ (flow rate $1.12 \mathrm{~mL} / \mathrm{min}$ ) allowing for interaction with cells.

The channel slide is then upside down inverted for $5 \mathrm{~min}$, then placed under the microscope and fluxed for 10 min with PBS to wash out any unspecifically adhered and easily detachable NDs. To image the NDs in fluorescence microscopy we took advantage of the intrinsic fluorescence of the NDs after conversion into the red form $\left(\lambda_{\text {ex }} 543 \mathrm{~nm}\right)$. Pictures corresponding to the five different fields of view are recorded after the washing. For image capturing and data analysis, Nikon NIS-Element AR software is used.

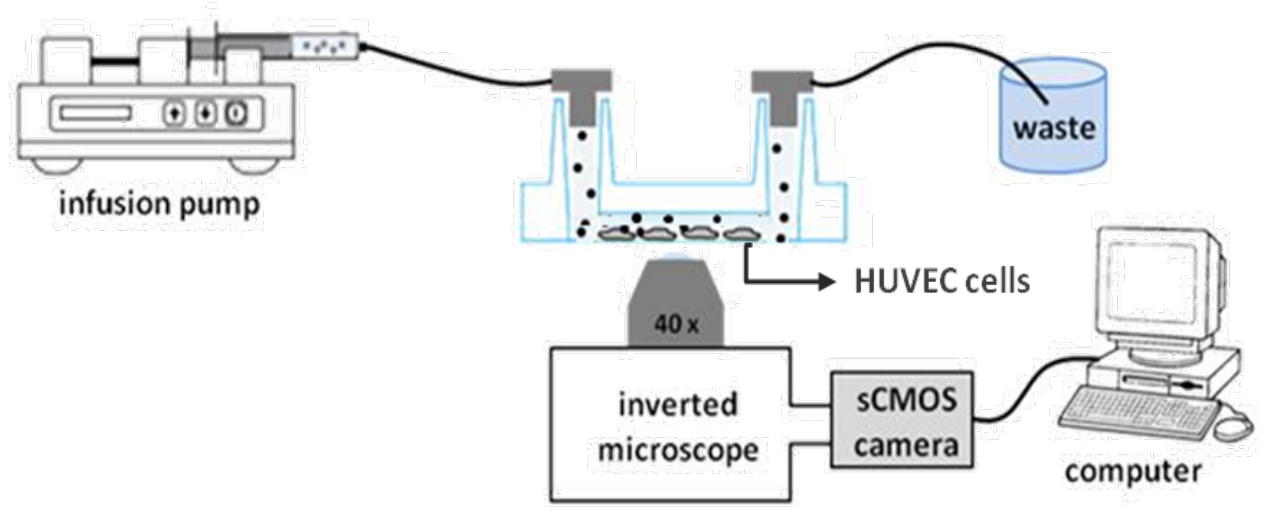

Scheme 2. In vitro cell targeting set-up 


\section{RESULTS AND DISCUSSION}

\section{Poly(PCDA)/PFC droplet formulation mechanism}

PCDA monomers can form vesicles with a bilayer arrangement in aqueous solutions with the polar heads oriented towards the water interface. ${ }^{44-48}$ In the case of PFC NDs, possible shell forming self-assembly of the PCDA units can result in a monolayer, triple layer or more, as described in Scheme S2 of the Supporting Information. Counting NDs with an average diameter larger than $800 \mathrm{~nm}$, detectable by bright field microscopy, the average surface occupied per PCDA molecules, i.e. 45 $\AA^{2}$, was determined by titrating the carboxyl moiety of the NDs shells (see Figure S1 of the Supporting Information). Considering the curvature of the NDs surface, this value is in line with the reported value, $26 \AA^{2}$, for Langmuir-Blodgett (LB) films of PCDA. ${ }^{49}$ An overcompression of a LB monolayer film by applying a surface pressure of $20 \mathrm{mM} / \mathrm{m}$ causes a film reorganization into a triple layer one with a molecular area of $7 \AA^{2} .^{50}$ Therefore, the molecular area of UV cured NDs measured in this work, although affected by a large error, up to $30 \%$, essentially due to the counting procedure, suggests that the PCDA shell of NDs is a monolayer analogous to LB films assembly.

Thus, the PCDA monolayer shells encapsulating a PFC liquid core in aqueous medium, are oriented with the lipophilic tail towards PFC, whereas the hydrophilic carboxylic head points towards the aqueous phase (see Scheme 3). PCDA shells can encapsulate different PFC in the liquid or in the gas state already available on the market and, among them, some are FDA approved. PFCs boiling points are found in a wide temperature range, depending on the carbon chain length and the fluorine/hydrogen ratio. Accordingly, PFCs can be liquid or gas in standard conditions, i.e. 1 atm. A milky PCDA/PFP droplets dispersion can be easily formed by sonicating a mixture of PCDA aqueous suspension and liquid PFP. However, to obtain stable droplets with PFB (b.p. $=-2{ }^{\circ} \mathrm{C}$ ), the latter needs to be liquefied under pressure or at very low temperatures. Sheeran et al. showed an interesting route to obtain PFB encapsulating droplets by condensing commercially available microbubbles, known as Micro-Marker ${ }^{\circledR}$ (VisualSonics, Canada). This was achieved by cooling the MBs and applying a pressure of $170 \mathrm{kPa} .^{51}$ Therefore, PFB NDs can be directly 
obtained once PFB is liquefied at low temperature under pressure, or both. In our case, the PFB was brought to liquid nitrogen temperature in order to limit the evaporation during further manipulations carried out at room temperature in closed vessel, as described in the Experimental Section. Additionally, the Laplace pressure contributes in stabilizing the nanodroplets by increasing the boiling temperature of PFB liquid core. Using the PFB Antoine's parameters, ${ }^{52}$ a boiling temperature of about $60^{\circ} \mathrm{C}$ is obtained when the external pressure is $3.4 \mathrm{~atm}$, corresponding to the vapor pressure of PFB at room temperature. This explains also why the majority of the NDs encapsulating PFB have diameter values around $250 \mathrm{~nm}$. Those with larger diameters, i.e. having less surface curvature, are less stable and most of them are bound to burst.

The photopolymerization of PCDA increases the stability of the formed NDs. During UV curing on the shell of the NDs, the hydrophobic tails of the PCDA molecules bear diacetylene moieties and are subjected to free radical polymerization. Strict PCDA molecular orientation is required to accomplish a topo-tactic sequence of alternating ene-yne bonds connecting transversally several PCDA tails, forming polymerized PCDA species of different lengths and molecular weights in the shell. The aqueous suspension of polymerized poly(PCDA)/PFC NDs is characterized by an intense blue color due to the conjugation of the double-triple bonds alternating sequences, whose intensity depends on the irradiation time and therefore on the polymerization degree. Moreover, the "blue" species can undergo a chromatic transition to a fluorescent red color upon heating, ${ }^{28}$ as illustrated in Scheme $\mathbf{3}$ and Figures $\mathbf{S 2}$ and S3 of the Supporting Information.

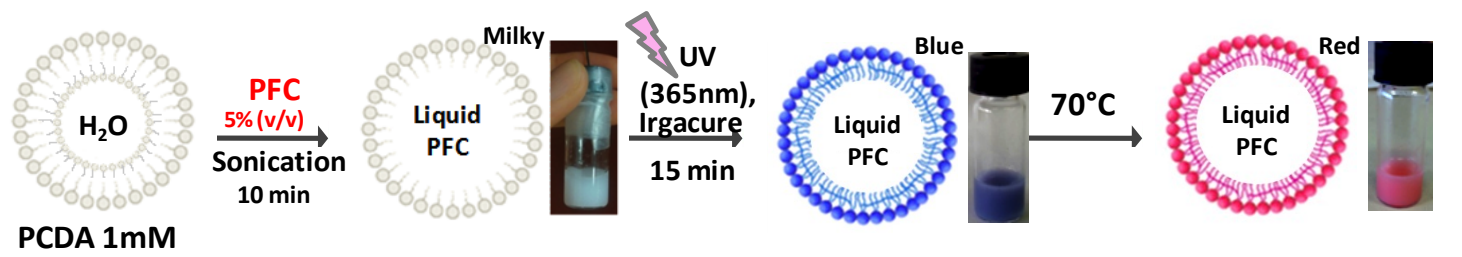

Scheme3. Poly(PCDA)/PFP nanodroplets formulation and chromatism. 


\section{Stability of poly(PCDA)/PFP and poly(PCDA)/PFB droplets:}

Both PFP and PFB poly(PCDA) shelled droplets exhibit a large size distribution in the submicron range of 200-1000 $\mathrm{nm}$, which can be tuned by centrifugation (see Figure S4 and Table S1 in the Supporting Information). However, for the PFB based droplets, in agreement with the findings reported by Sheeran et al. only those with a size below $500 \mathrm{~nm}$ remain stable once subjected to atmospheric pressure and RT. ${ }^{53}$ Average sizes of PFB and PFP encapsulating NDs, are monitored by DLS and reported as a function of time in Figure 1 (a). Sizes below $600 \mathrm{~nm}$ for both PFP and PFB NDs stay constant for more than a month, while the larger sizes in the case of PFP NDs increase by a factor of two within the first five days. NDs counts, estimated by optical microscopy over the same period of time, show as indicated in Figure $\mathbf{1}$ (b) a pronounced decrease for poly(PCDA)/PFP of the NDs number, occurring again in the first five days. The time evolution of the NDs highlights a good NDs stability over a month, with a size increase and a numerical concentration drop occurring in the first five days. The lower stability of NDs with an initial larger size could be explained by a limited polymerization of PCDA, due to the larger distance separating the PCDA molecules as consequence of a lower density of monomers over the larger PFP core.
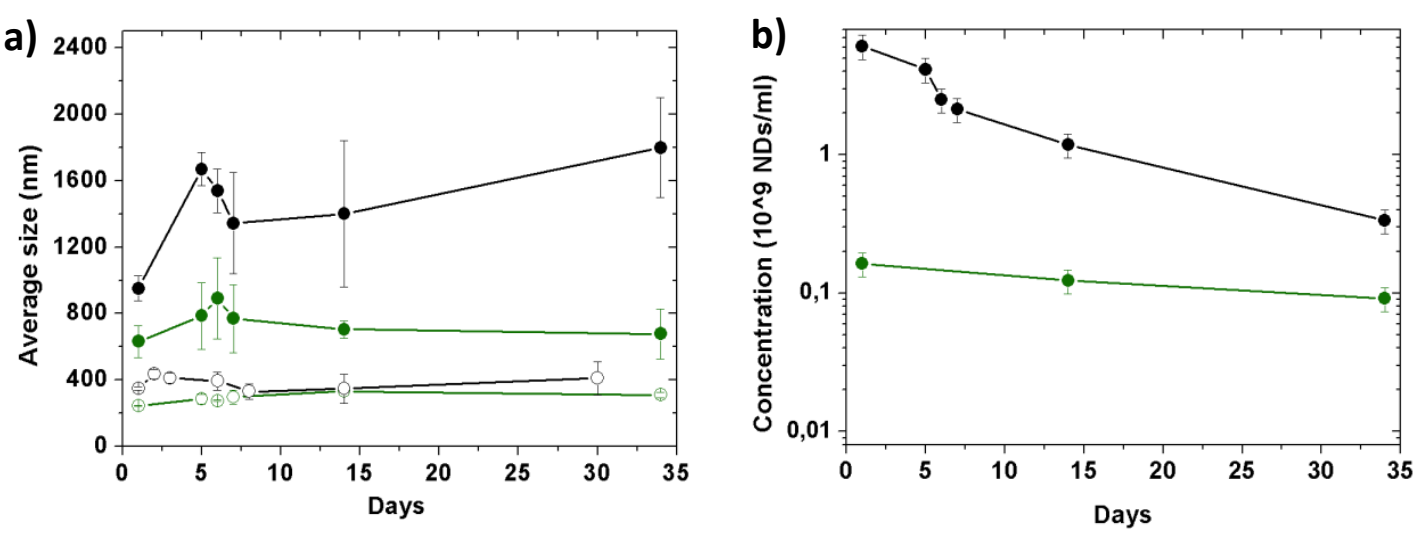

Figure 1. a) Average size variation of poly(PCDA)/PFP (black) and poly(PCDA)/PFB (green) over time by DLS, the filled and empty symbols represent NDs with initially larger and smaller size respectively; b) poly(PCDA)/PFP (black) and poly(PCDA)/PFB (green) concentration over time (The lines represent a guide for the eyes). Fractions of NDs with selected dimensions are obtained by separating pellet and supernatant, respectively upon centrifugation at (3200 rpm/973 g-force) for $4 \mathrm{~min}$. 
Moreover poly(PCDA)/PFC samples exhibit strong negative $Z$ potential, stabilizing the colloidal system, with no significant difference for both blue and red forms (-32 \pm 2 $\mathrm{mV}$ and $-33 \pm 2 \mathrm{mV}$, respectively). Therefore, the heat treatment maintains the ionization of the carboxyl moiety at the poly(PCDA) shell surface unchanged, suggesting that the chromatic transition is attributed to minor structural variations limited to the orientation of the carboxyl groups.

\section{Poly(PCDA)/PFC NDs shell characterization by GPC and MALDI-ToF}

The degree of polymerization of the shell of poly(PCDA)/PFC NDs is investigated by GPC and MALDI-ToF for samples treated with different UV curing times. Figure 2 shows the MW distributions obtained for poly(PCDA) shells with UV curing of $5 \mathrm{~min}$ and $15 \mathrm{~min}$, respectively, in comparison to the PCDA monomer. The chromatograms display a higher peak at around $5800 \mathrm{Da}$ and a lower peak at $15500 \mathrm{Da}$ corresponding to a polymerization degree of 16 and 41 , respectively.

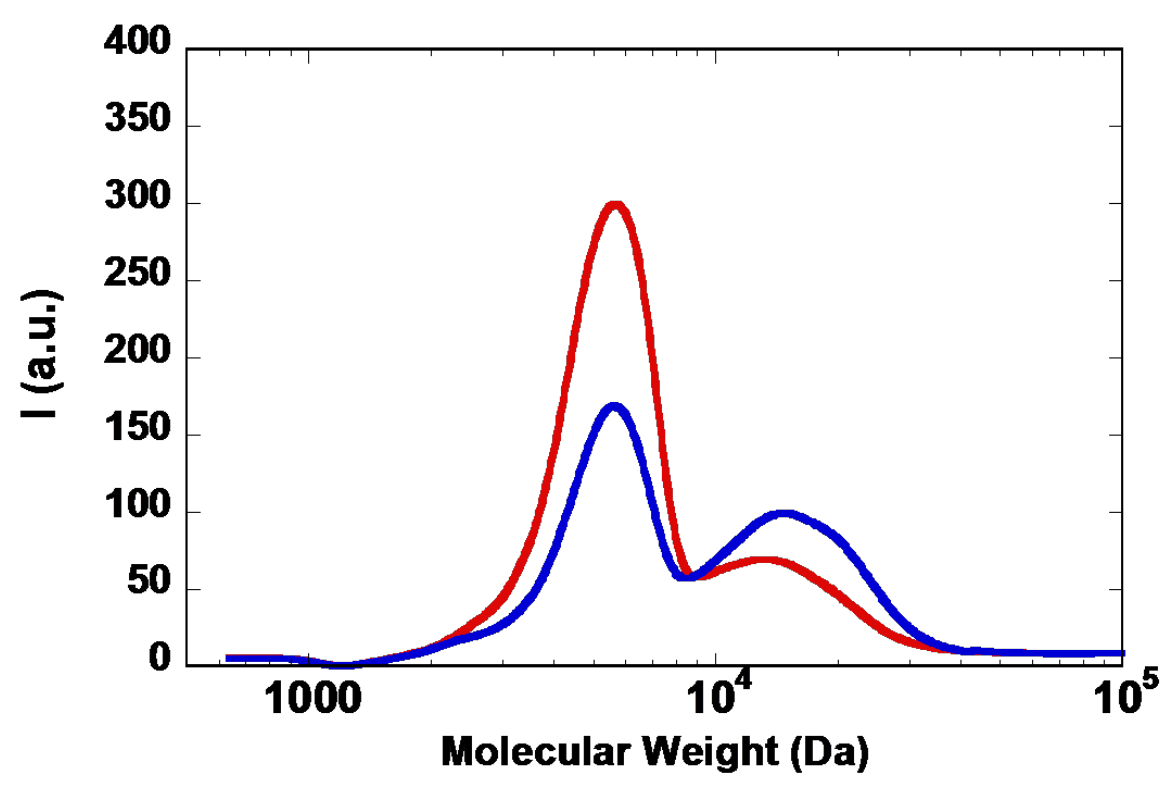

Figure 2. Molecular weight distribution of freeze-dried NDs polymerized for $5 \mathrm{~min}$ (red) and for $15 \mathrm{~min}$ (blue). Chromatograms were normalized to the area of the peaks.

The UV curing time has a deep influence on the chromatogram shapes, changing the relative heights of the two peaks. The intensity of the peak of the low molecular weight species decreases when the curing time changes from 5 to $15 \mathrm{~min}$, whereas 
the opposite behavior is observed for the high molecular weight peak. Moreover, a shoulder is observed at around 20,000 Da, highlighting the presence of higher molecular weight species. These findings confirm that the UV triggered radical polymerization is responsible for the polymerization of PCDA and that the time frame characteristic of this reaction covers several minutes. MALDI-ToF based mass analyses confirm the GPC results. Spectra are recorded after re-dispersing poly(PCDA)/PFC samples in acetate buffer (positive mode) and in phosphate buffer (negative mode). Combinatorial analysis performed to quantify the primary MALDIToF MW-distributions, indicates molecular weights as high as those found by the GPC analysis as described Figure $\mathbf{3}$ and Figure S5 in the Supporting Information.

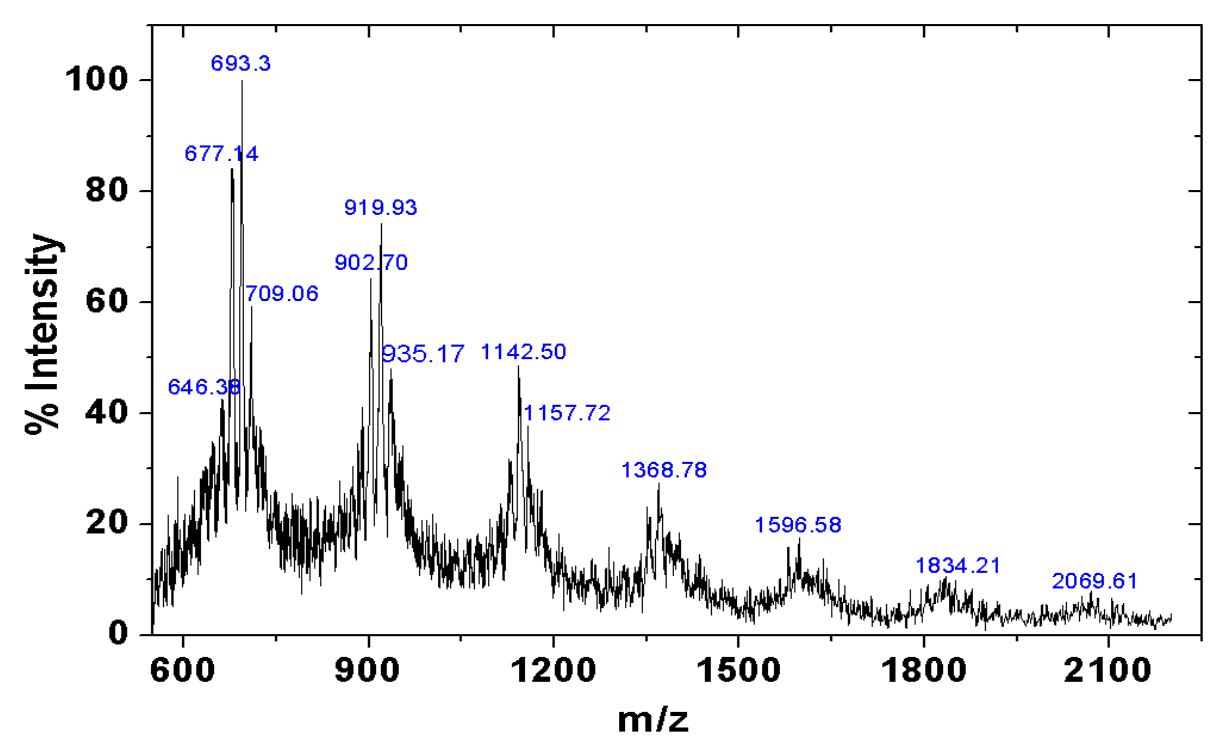

Figure 3. Representative MALDI-ToF spectrum obtained in negative mode for poly(PCDA)/PFC NDs polymerized under UV irradiation for 15 min dispersed in phosphate buffer in the presence of dithranol as matrix reagent.

Based on the chemical nature of the poly(PCDA) chains investigated in this study, a multiple charging process is likely to occur in acetate buffer by multiple addition of protons and sodium cations or, in phosphate buffer, by multiple deprotonation combined with a partial charge compensation by proton and sodium addition, which should explain the low mass/charge values reported in Figure 3 and Figure S5. Moreover, it can be assumed that the rather hydrophobic nature of the poly(PCDA) chains allowed for observation of poly(PCDA)-dithranol complexes. Significant results of the combinatorial analyses are summarized in Tables S2 and S3 of the Supporting 
Information for negative and positive mode, respectively. In this analysis, also the mentioned possible stacking complexes between the poly(PCDA) and the matrix molecules have been considered. Structural representations of selected identified polydiacetylene species are illustrated in Scheme $\mathbf{S 4}$ of the Supporting Information. Such analysis suggests that high degrees of polymerization are obtained when polymerizing PCDA by UV curing. The identified polymer sizes fit in the bulk of the chromatogram of Figure 2, confirming a bimodal distribution of the poly(PCDA) molecular weights with a prevailing peak at around $8000 \mathrm{Da}(\mathrm{DP}=22$ ) and a minor peak at about $15000 \mathrm{Da}(\mathrm{DP}=39)$. According to our GPC and MALDI-ToF results, about 40 PCDA molecules have been polymerized by UV curing organized in patches of surfactant crosslinked molecules with a 2D size of about $200 \mathrm{~nm}^{2}$.

\section{Acoustic droplets vaporization (ADV) of poly(PCDA)/PFC}

ADV experiments are carried out in vitro at $37^{\circ} \mathrm{C}$, with nanodroplets dispersed in human serum in order to mimic as much as possible the physiological conditions. Indeed, the presence of proteins in blood serum such as albumin lowers the surface tension of droplets and therefore impacts favorably on the ADV efficiency by rendering the process more efficient. Droplets of encapsulated PFP or PFB required different conditions to achieve ADV, according to their different boiling points. In our tests, US intensities of $3.6 \mathrm{~W} / \mathrm{cm}^{2}$ and $0.5 \mathrm{~W} / \mathrm{cm}^{2}$ are applied on the PFP and PFB NDs, respectively. The most direct evidence supporting the occurrence of the liquid $\leftrightarrow$ vapor transition of the core is the observation of an abrupt change of size of the insonified NDs, passing from submicron to MBs with diameters of few microns. Micrographs in Figures $4 \mathrm{a}$ and $4 \mathrm{c}$ show that, before US activation, both PFP and PFB NDs are hardly detectable in bright field modality, as their size is below or at the most approaching the resolution limit of the microscope. 
a)

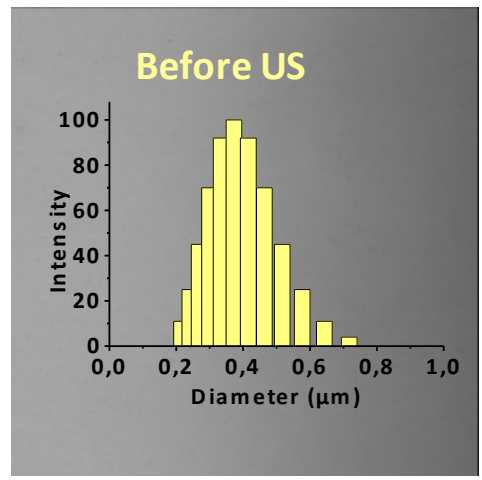

c)

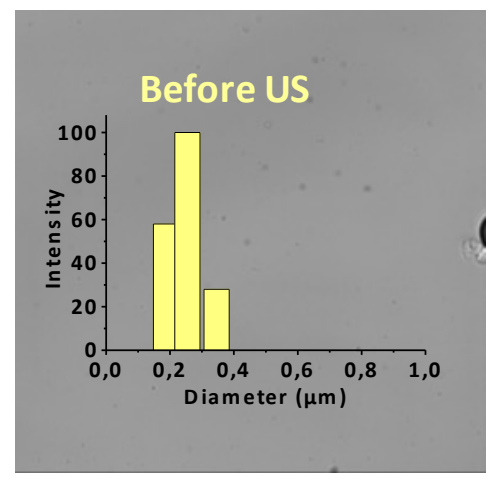

b)

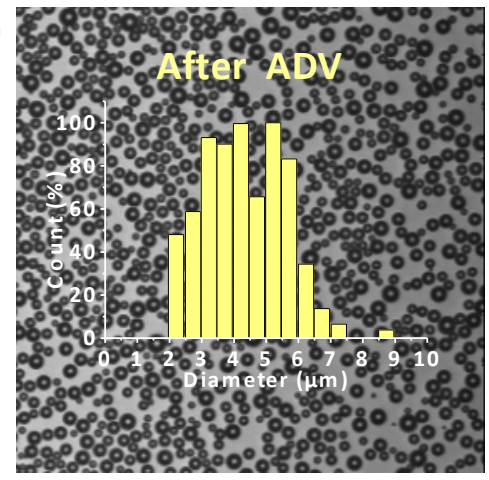

d)

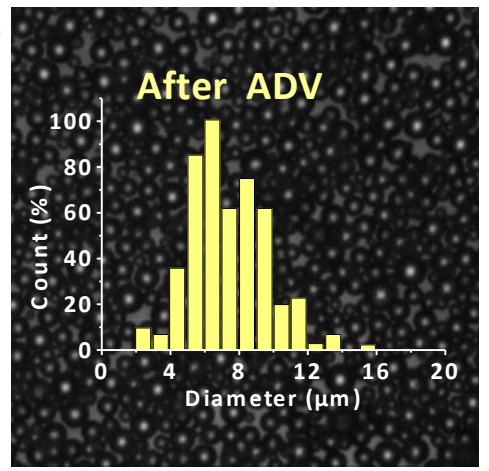

Figure 4. Poly(PCDA)/PFP nanodroplets before, a), and after, b), ADV at $3.6 \mathrm{~W} / \mathrm{cm}^{2}$, respectively; poly(PCDA)/PFB nanodroplets before, c), and after, d), ADV at $0.5 \mathrm{~W} / \mathrm{cm}^{2}$, respectively. Micrographs $b$ ) and d) were taken after $10 \mathrm{~min}$ from the US activation.

Moreover, in the same region of interest, ROI, or in different zones of the channel micron sized particles, i.e. MBs, were not visualized. This finding confirms the stability of the NDs and rules out the hypothesis of a spontaneous phase transition process slowly progressing during the observation. After US irradiation a relevant number of MBs appears (see Figures $4 b$ and $4 d$ ). The size of the ADV generated MBs does not exceed $10 \mu \mathrm{m}$ in size as required for a systemically injectable device. The average size of the MBs depends on the size of the parent NDs, but also on the PFC core, as highlighted in Table 1. After ADV the average size of poly(PCDA)/PFP NDs increased by a factor of 10 , whereas poly(PCDA)/PFB MBs displayed an average size 30-fold larger than the parent NDs. 
Table 1. Poly(PCDA)/PFC size before and after ADV

\begin{tabular}{lcc}
\hline Type & $\begin{array}{c}\text { NDs diameter }(\mathrm{nm}) \\
\text { [before ADV] }\end{array}$ & $\begin{array}{c}\text { MBs diameter }(\mu \mathrm{m}) \\
\text { [after ADV] }\end{array}$ \\
\hline Poly(PCDA)/PFP & $400 \pm 20$ & $5 \pm 1$ \\
& $700 \pm 120$ & $8 \pm 3$ \\
Poly(PCDA)/PFP “red” & $760 \pm 100$ & $9.4 \pm 2.7$ \\
Poly(PCDA)/PFB & $250 \pm 10$ & $7.5 \pm 2.7$ \\
\hline
\end{tabular}

It was reported that ADV promotes a size increase of about 5- to 6-fold over milliseconds time lapse for phospholipidic NDs, in agreement with simple considerations based on ideal gas. ${ }^{45,46}$ However, MBs size has a complex dependence, not yet completely elucidated, ${ }^{47}$ on medium, perfluorocarbon characteristics, shell type. ${ }^{47}$ Time is a factor to take into account in analyzing NDs $\leftrightarrow$ MBs transition. Reznik et al. report on $400 \mathrm{~nm}$ PFC encapsulating droplets which expand into MBs with a diameter of $1.4 \mu \mathrm{m}$ after $1 \mathrm{~ms}$ and $5.6 \mu \mathrm{m}$ after $1 \mathrm{~s}$ from $A D V .{ }^{54}$ Sheeran et al. have shown that there is a significant difference on the size increase between degassed and undegassed samples upon ADV, suggesting that undegassed samples tend to increase more in size over the time, ${ }^{48}$ excluding a coalescence effect between MBs. An increase of about 25 times of the particle diameter, after ADV, is reported for droplets of PFP stabilized in a suspension of saline containing bovine serum albumin. This further expansion was explained by accounting for an uptake of gasses from the air-saturated host medium into the MBs. ${ }^{55}$ These conditions apply also to our experiments, performed in undegassed medium containing human serum to mimic as much as possible the physiological conditions. Our results indicate that the behavior of the microbubbles after ADV depends on the boiling point of the liquid core of the parent nanodroplets. If the latter is superheated (as in the case of PFB), the microbubble lifetime could be surprisingly long. It has been reported that lipid coated microbubbles with a size range of $1-5 \mu \mathrm{m}$, encapsulating air lasted for up to one year dispersed in degassed water. ${ }^{56}$ Such prolonged lifetime was attributed to the surfactant coating of the MBs due to the shell resistance to gas permeation. In 
our systems the stability conditions are more favorable than in the reported example, due to the marked hydrophobicity of the encapsulated gas (more than the air), resulting in a very little tendency to diffuse out in an aqueous environment. No evidences of bursts or inertial cavitation, IC, in the form of broken shells or debris were observed after US irradiation. ADV proceeded without accompanying IC events, in agreement with studies reporting that the thresholds of these two processes are not governed by the same parameters nor have the same dependence from the US frequency of irradiation. ${ }^{57}$

\section{Acoustic attenuation of poly(PCDA)/PFC NDs}

The acoustic attenuation measurements of nanodroplets before and after irradiation with US at $1 \mathrm{MHz}$ were performed at $37^{\circ} \mathrm{C}$, in a degassed cell culture medium containing FBS. Figure 5 a shows that the attenuation of PFP NDs measured at $1 \mathrm{MHz}$ increases by increasing the intensity of the applied US up to $3.6 \mathrm{~W} / \mathrm{cm}^{2}$. Whereas the poly(PCDA)/PFB NDs attenuation reaches a maximum when the power of the incident US irradiation is only $0.5 \mathrm{~W} / \mathrm{cm}^{2}$, consistently with the lower boiling point of PFB $\left(-2{ }^{\circ} \mathrm{C}\right)$. The dramatic change of attenuation before and after US irradiation indicates that the process is a liquid-vapor transition and not an aggregation/coalescence effect where the absence of a phase transition could not justify such a remarkable attenuation increase. Moreover, the poly(PCDA)/PFP NDs do not exhibit any acoustic response before their activation, even at the physiological temperature of $37^{\circ} \mathrm{C}$. The behavior of the poly(PCDA)/PFB NDs, which show a very low, but detectable acoustic attenuation (see Figure $\mathbf{5 b}$ ), is attributed to a small fraction of larger PFB droplets with the core partially or already completely vaporized, although not detected in bright field microscopy. This is supported by the attenuation spectra of PFB encapsulated NDs without US activation as a function of the time. The results reported in Figure $\mathbf{S 6}$ of Supporting Information show that attenuation is absent for NDs with small size (300 nm), suggesting that no spontaneous vaporization occurred. PFB NDs with larger size (700 nm as average) display vaporization as indicated by the low, but not negligible attenuation recorded within $1 \mathrm{~min}$. It is worth noticing that the attenuation spectra after ADV for both 
types of NDs, i.e. the PFP and PFB based poly(PCDA), reveal an acoustic resonance between 1 and $2 \mathrm{MHz}$ similar to the peak resonance of lipid shelled MBs. ${ }^{58-61}$
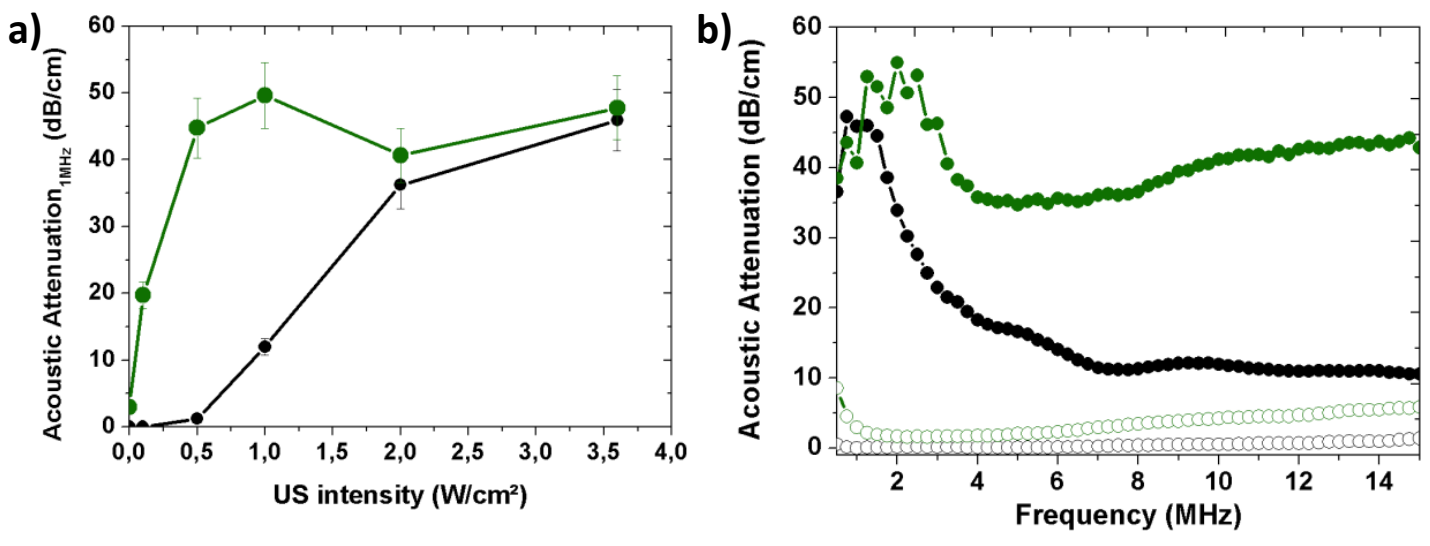

Figure5. a) Acoustic attenuation at $1 \mathrm{MHz}$ as a function of applied US intensity for poly(PCDA)/PFP (black) and poly(PCDA)/PFB (green); b) Acoustic attenuation spectra of poly(PCDA)/PFP (black) and poly(PCDA)/PFB (green) nanodroplets before (empty markers) and after ADV (filled markers) at $3.6 \mathrm{~W} / \mathrm{cm}^{2}$ and $0.5 \mathrm{~W} / \mathrm{cm}^{2}$, respectively (lines are a guide for the eyes).

\section{Cytotoxicity evaluation of poly(PCDA)/PFC droplets:}

Many studies reported the use of PCDA for drug delivery applications in the form of liposomes or monolayer films for biosensing applications due to its optical features and sensitivity to external stimuli once polymerized. ${ }^{32,} 62$ Hybrid systems incorporating PCDA have been investigated for their cytotoxicity. However, as far as we know, no data are available concerning the impact of the poly(PCDA) combined with PFCs on living tissues. Studying the viability of NIH 3T3 fibroblasts after $24 \mathrm{~h}$ of incubation with different ND concentrations, we demonstrate the biocompatibility of poly(PCDA). The test is performed only on PFP based droplets since NDs encapsulating PFB do not allow viability tests at high concentrations at $37{ }^{\circ} \mathrm{C}$ for 24 hours, due to their limited stability in these conditions. Figure 6 shows that, within the error of MTT tests, no significant decrease in cells viability occurs, when compared with the control even for the highest amount estimated as $5 \times 10^{8}$ droplets $/ \mathrm{mL}$. 
a)
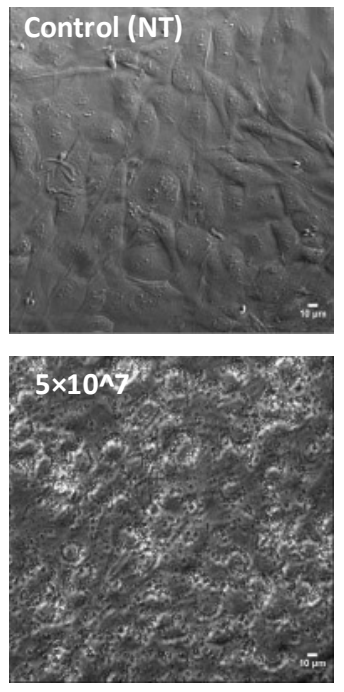

b)

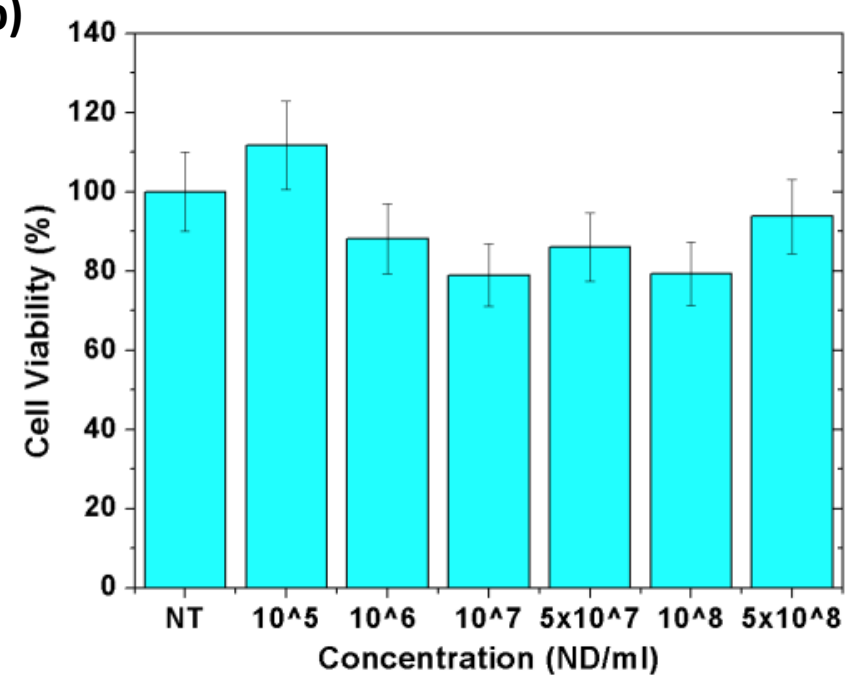

Figure 6. Cytotoxicity evaluation of poly(PCDA)/PFP droplets by MTT tests on fibroblasts NIH 3T3: a) control cells (in the absence of poly(PCDA)/PFP NDs) and cells exposed to an indicative amount of $5 \times 10^{7} \mathrm{NDs}$; b) cells viability histogram as a function of droplets concentration.

\section{Poly(PCDA)/PFP NDs surface modifications}

\section{Functionalization with fluoresceinamine}

The presence of carboxyl groups as polar head exposed on the surface of PCDA/PFC NDs offers the possibility to tailor the chemistry at the water interface of the construct after UV curing and allows for easy surface modifications to implement their functionalities by attaching ligand molecules, small dyes, nanoparticles, etc. ${ }^{63-65}$ Molecules with primary amine groups can be coupled to the carboxyl groups of PCDA NDs in aqueous media via zero length spacer EDC/NHS chemistry. As a proof of concept, the fluoresceinamine dye is conjugated to poly(PCDA)/PFP NDs for labeling the shell (see Scheme S3 in the Supporting Information). The resulting NDs appear as single fluorescent green pixels when excited with an $\mathrm{Ar}^{+}$laser at $488 \mathrm{~nm}$. The shell modification did not hinder ADV for converting the NDs into MBs, as highlighted in Figure $7 d$. It can be noticed that the fluorescence emission decreases due to both the expansion of the shell and the corresponding decrease of the fluorophore density; visualization is nevertheless possible increasing the gain (see Figure 7). 


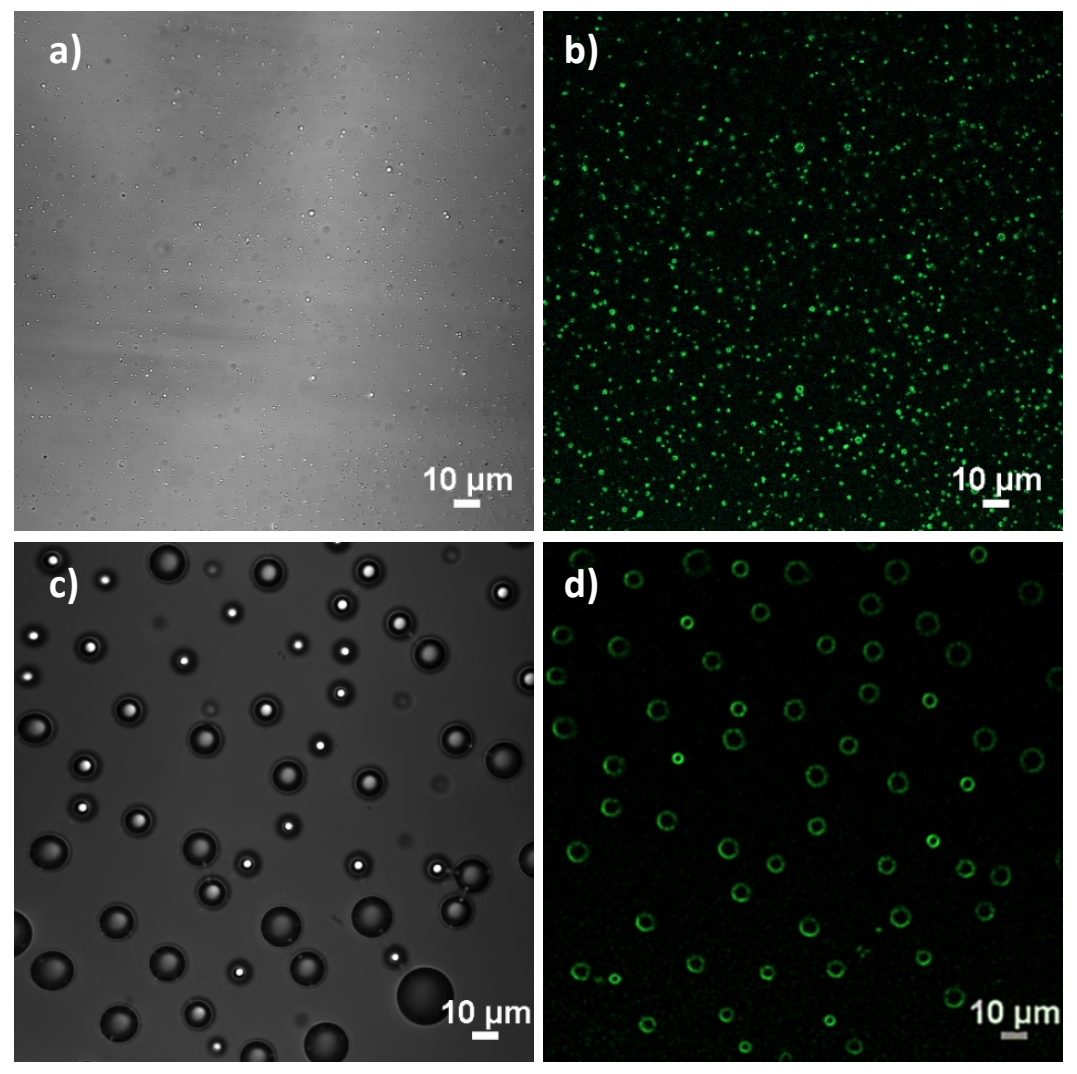

Figure 7. Poly(PCDA)/PFP-Fluoresceinamine before ADV: a) and b) transmission microscopy and confocal microscopies, respectively. After ADV: c) and d) transmission and confocal microscopies, respectively. In d) gain was increased from 145 to 150 (pinhole: small-30 $\mu \mathrm{m}$ ).

\section{Functionalization with cyclo-RGDand in vitro HUVEC cell targeting}

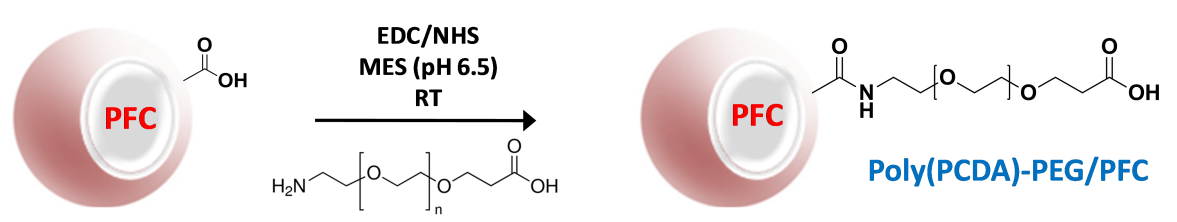

O-(2-Aminoethyl)- $O^{\prime}$-(2-carboxyethyl)PEG

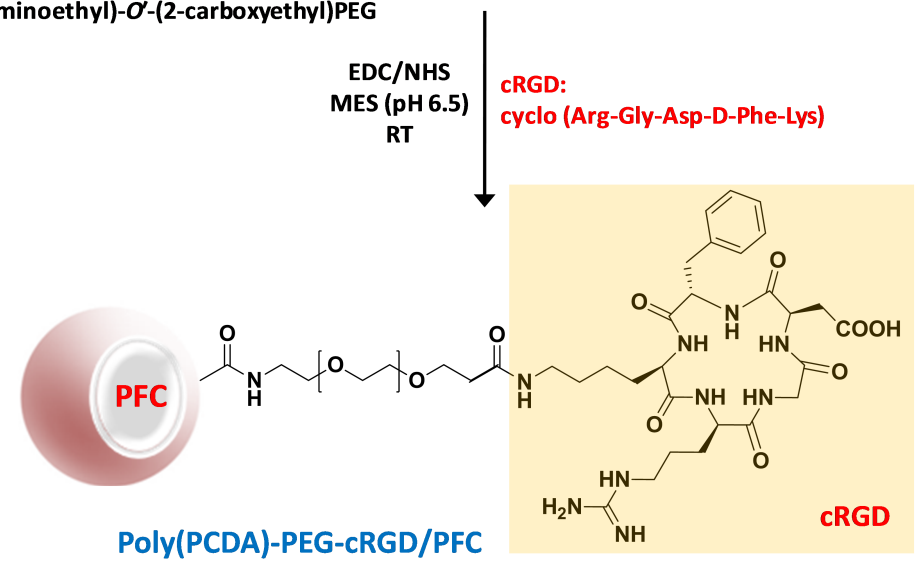

Scheme 4. PEGylation and conjugation with CRGD peptide of poly(PCDA)/PFC NDs. 
Various peptides can provide specific interactions with cells and promote bioadhesion by recognition of specific receptors present on target sites. RGD is a sequence of arginine, glycine and aspartic acid, commonly known for targeting $\alpha_{v} \beta_{3}$ integrins over-expressed in tumor cells during angiogenesis. ${ }^{66-69} \mathrm{~A}$ pentapeptide, which includes the RGD sequence followed by a D-Phe-Lys moiety to close the cyclic structure, i.e. cyclo (Arg-Gly-Asp-D-Phe-Lys) or CRGD, has been chosen in order to enable tethering onto the surface of the poly(PCDA) NDs via EDC/NHS chemistry, as described in Scheme 4. In this study we are focused on the biointerface properties of the NDs as this is the form designed to target and tether pathological cells. It should be considered that, in vivo, a factor which interferes with the tethering of NDs to cells is the build-up of a plasma protein corona on the NDs shell once the construct is injected in the blood pool. ${ }^{66}$ We found a marked adhesion of NDs without CRGD functionalization on HUVECs. Another factor perturbing the efficacy of the active targeting of integrins rich cells is the high density of carboxyl groups on the NDs surface, which can promote a strong, although non-specific, tendency to interact with cells. The presence of such additional interaction with cells masks and competes with the efficiency of the active CRGD promoted targeting of integrins receptors. In an effort to prevent or limit the establishment of a protein corona and the non-specific targeting, CRGD has been tethered to the surface of the poly(PCDA) NDs via a PEG spacer to facilitate the spatial orientation of CRGD by the enhanced linker flexibility and to increase the distance of the CRGD from the NDs surface to avoid non-specific interactions of the polar heads of PCDA with the cell membrane and to place the CRGD ligand molecules above any potential protein corona.

Figure 8 illustrates the bioadhesion study in dynamic conditions, i.e. steadily perfusing the suspension of targeted and PEGylated "red" form of poly(PCDA) NDs through microchannels in which HUVEC cells have been previously seeded and brought to confluence. Pegylation is an efficient method to limit the unspecific interactions of NDs with HUVEC cells, promoted by the presence on the shells of a large number of carboxyl groups. ${ }^{71}$ NDs, converted into the red fluorescent form for immediate optical and fluorescence microscopy detection without the addition of any external fluorescence labels, appear as "red pixels" due to the ND dimensions below the microscope optical resolution. Comparison on the number of bioadhered 
pegylated NDs with and without RGD modification over the HUVEC cells cultured in the microchannel slide suggests an enhanced targeting specificity of RGD modified NDs surfaces. The channel slide was then inverted to detach the weakly bound NDs by washing them out with PBS. The persisting cell-bound NDs, considered as the fraction of particles specifically interacting with the cells surface, were subsequently counted and results were normalized to the number of cells present in the ROI. With CRGD conjugated to the surface via PEG spacer, the bioadhesion of NDs is significantly enhanced $(0.6 \pm 0.15 \mathrm{ND} /$ cell) as compared to the plain poly(PCDA)PEG/PFP NDs used as control (0.25 $\pm 0.06 \mathrm{ND} /$ cell). The specificity of bioadhesion to endothelial cells decreases in the case of non-PEGylated poly(PCDA)-cRGD NDs (see Figure $\mathbf{S 6}$ in the Supporting Information). As anticipated, the enhanced targeting efficacy and specificity can be attributed to the flexibility of PEG chains hindering non-specific bindings and bringing the CRGD moiety attached to the PEG chain end over the accumulated protein corona. These results are promising in the general perspective of an active targeting of poly(PCDA) NDs based on ligand-receptor interaction 

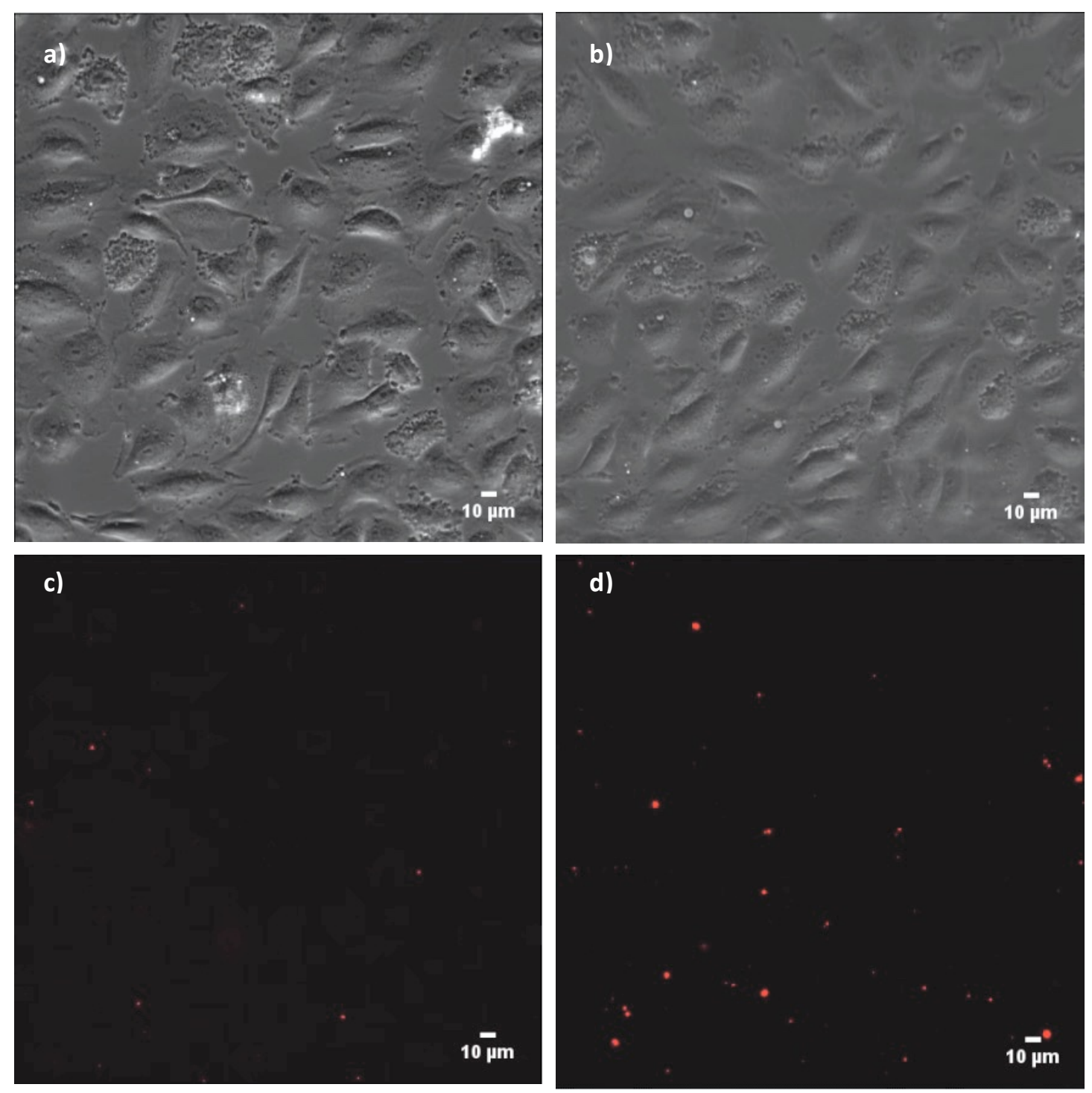

Figure 8. Adhesion of pegylated poly(PCDA) NDs to HUVEC. Micrographs (40x) after $10 \mathrm{~min}$ NDs flowing, $5 \mathrm{~min}$ inversion and $10 \mathrm{~min}$ PBS washing: a) and c) cells treated with poly(PCDA)-PEG/PFP NDs (control) in bright field and fluorescence microscopy, respectively; b) and d) cells treated with poly(PCDA)PEG-cRGD/PFP in bright field and fluorescence microscopy, respectively.

\section{CONCLUSION}

Polymerized PCDA nanodroplets encapsulating low boiling point perfluorocarbon liquids represent an hybrid construct intermediate between lipid and polymer shelled NDs, combining the advantages of both classes of NDs. In this exploratory study optical, acoustic and ADV properties of this assembly were evaluated as well as cytoxicity and targeting efficacy toward endothelial angiogenic cells. UV curing of the PCDA shell can involve more than 40 diacetylenic molecules on the shell although a study of the factors influencing the radical polymerization, i.e. droplet size, surface 
curvature as well as curing time, has to be specifically addressed in a future work. We showed that the curing provides a good colloidal stability with both encapsulated PFP and PFB introducing $200 \mathrm{~nm}^{2}$ patches of 40 polymerized PCDA molecules, dispersed on a monolayer of unbound PCDA molecules. As liquids these perfluorocarbons make the particles active for magnetic resonance detection and represent a reservoir where hydrophobic drug molecules could be dissolved, opening the possibility to used the poly(PCDA) NDs for targeted drug delivery. The same particles once converted into MBs by means of ADV, behave as US contrast agents supporting a dual imaging modality. These results indicate a promising system on the basis of self-assembled and subsequently polymerized diacetylenic particles capable of penetrating the biological barriers based on the nano size of the droplets and the US triggered liquid $\leftrightarrow$ gas transition. The characteristics indicate a good potential for the assembly for imaging and drug delivery applications. ADV based systems revert the use of ultrasounds, so far considered almost exclusively the carrier of the diagnostic information. As consequence the transformed droplets allow for several options, from already known imaging capability to transport of drugs dissolved in the hydrophobic core of the particles, localized in a tissue area presenting pathology. Formulation of small nanodroplets, smaller than the ones reported here, is possible by tuning the vapor pressure of the PFC core liquid, stirring speed or sonication power. With such systems made available the crossing of biological barriers will be feasible and delivery of drug more localized and powerful.

Whether the core phase transition could be triggered by other non-mechanical excitations, as for example by ionizing radiation has to be investigated. Such possibility would open the road to the use of NDs as injectable and tumor addressable in situ dosimetric device for personalized medicine.

\section{ACKNOWLEDGMENTS}

This work has been partly supported by EU H2020 "AMPHORA", contract n. 766456 


\section{REFERENCES}

(1) Dollet, B.; Meer, S. M. van der; Garbin, V.; Jong, N. de; Lohse, D.; Versluis, M. Nonspherical Oscillations of Ultrasound Contrast Agent Microbubbles. Ultrasound Med. Biol. 2008, 34 (9), 1465-1473.

(2) Lee, H. J.; Yoon, T.-J.; Yoon, Y. I. Synthesis of Ultrasound Contrast Agents: Characteristics and Size Distribution Analysis (Secondary Publication). Ultrason. Ultrason. 2017, 36 (4), 378-384.

(3) Unnikrishnan, S.; Klibanov, A. L. Microbubbles as Ultrasound Contrast Agents for Molecular Imaging: Preparation and Application. Am. J. Roentgenol. 2012, 199 (2), 292-299.

(4) Stride, E.; Saffari, N. Microbubble Ultrasound Contrast Agents: A Review. Proc. Inst. Mech. Eng. [H] 2003, 217 (6), 429-447.

(5) Dijkmans, P. A.; Juffermans, L. J. M.; Musters, R. J. P.; van Wamel, A.; ten Cate, F. J.; van Gilst, W.; Visser, C. A.; de Jong, N.; Kamp, O. Microbubbles and Ultrasound: From Diagnosis to Therapy. Eur. J. Echocardiogr. 2004, 5 (4), $245-246$.

(6) Sirsi, S. R.; Borden, M. A. Microbubble Compositions, Properties and Biomedical Applications. Bubble Sci. Eng. Technol. 2009, 1 (1-2), 3-17.

(7) Capece, S.; Domenici, F.; Brasili, F.; Oddo, L.; Cerroni, B.; Bedini, A.; Bordi, F.; Chiessi, E.; Paradossi, G. Complex Interfaces in "Phase-Change" Contrast Agents. Phys. Chem. Chem. Phys. 2016, 18 (12), 8378-8388.

(8) Capece, S.; Chiessi, E.; Cavalli, R.; Giustetto, P.; Grishenkov, D.; Paradossi, G. A General Strategy for Obtaining Biodegradable Polymer Shelled Microbubbles as Theranostic Devices. Chem. Commun. 2013, 49 (51), 5763-5765.

(9) Oddo, L.; Cerroni, B.; Domenici, F.; Bedini, A.; Bordi, F.; Chiessi, E.; Gerbes, S.; Paradossi, G. Next Generation Ultrasound Platforms for Theranostics. J. Colloid Interface Sci. 2017, 491, 151-160.

(10) Grishenkov, D.; Pecorari, C.; Brismar, T. B.; Paradossi, G. Characterization of Acoustic Properties of PVA-Shelled Ultrasound Contrast Agents: Linear Properties (Part I). Ultrasound Med. Biol. 2009, 35 (7), 1127-1138.

(11) Abou-Saleh, R. H.; Swain, M.; Evans, S. D.; Thomson, N. H. Poly(Ethylene Glycol) Lipid-Shelled Microbubbles: Abundance, Stability, and Mechanical Properties. Langmuir 2014, 30 (19), 5557-5563.

(12) Bjerknes, K.; Sontum, P. C.; Smistad, G.; Agerkvist, I. Preparation of Polymeric Microbubbles: Formulation Studies and Product Characterisation. Int. J. Pharm. 1997, 158 (2), 129-136.

(13) Lentacker, I.; De Geest, B. G.; Vandenbroucke, R. E.; Peeters, L.; Demeester, J.; De Smedt, S. C.; Sanders, N. N. Ultrasound-Responsive Polymer-Coated Microbubbles That Bind and Protect DNA. Langmuir 2006, 22 (17), 7273-7278.

(14) Prajapati, B. G. A Conceptual Review on Micro Bubbles. Biomed. J. Sci. Tech. Res. 2017, 1 (2), 353-359.

(15) Hernandez, C.; Gulati, S.; Fioravanti, G.; Stewart, P. L.; Exner, A. A. Cryo-EM Visualization of Lipid and Polymer-Stabilized Perfluorocarbon Gas Nanobubbles - A Step Towards Nanobubble Mediated Drug Delivery. Sci. Rep. 2017, 7.

(16) Martin, K. H.; Dayton, P. A. Current Status and Prospects for Microbubbles in Ultrasound Theranostics: Current Status and Prospects for Microbubbles. Wiley Interdiscip. Rev. Nanomed. Nanobiotechnol. 2013, 5 (4), 329-345. 
(17) Kothapalli, S. V. V. N.; Wiklund, M.; Janerot-Sjoberg, B.; Paradossi, G.; Grishenkov, D. Investigation of Polymer-Shelled Microbubble Motions in Acoustophoresis. Ultrasonics 2016, 70, 275-283.

(18) Lin, C.-Y.; Pitt, W. G. Acoustic Droplet Vaporization in Biology and Medicine. BioMed Research International 2013.

(19) Sheeran, P. S.; Matsuura, N.; Borden, M. A.; Williams, R.; Matsunaga, T. O.; Burns, P. N.; Dayton, P. A. Methods of Generating Submicrometer Phase-Shift Perfluorocarbon Droplets for Applications in Medical Ultrasonography. IEEE Trans. Ultrason. Ferroelectr. Freq. Control 2017, 64 (1), 252-263.

(20) Sheeran, P. S.; Luois, S.; Dayton, P. A.; Matsunaga, T. O. Formulation and Acoustic Studies of a New Phase-Shift Agent for Diagnostic and Therapeutic Ultrasound. Langmuir 2011, 27 (17), 10412-10420.

(21) Zhou, Y. Application of Acoustic Droplet Vaporization in Ultrasound Therapy. $J$. Ther. Ultrasound 2015, 3 (1).

(22) Astafyeva, K.; Somaglino, L.; Desgranges, S.; Berti, R.; Patinote, C.; Langevin, D.; Lazeyras, F.; Salomir, R.; Polidori, A.; Contino-Pépin, C.; et al. Perfluorocarbon Nanodroplets Stabilized by Fluorinated Surfactants: Characterization and Potentiality as Theranostic Agents. J. Mater. Chem. B 2015, 3 (14), 2892-2907.

(23) Rapoport, N. Phase-Shift, Stimuli-Responsive Perfluorocarbon Nanodroplets for Drug Delivery to Cancer: Phase-Shift Perfluorocarbon Nanoemulsions. Wiley Interdiscip. Rev. Nanomed. Nanobiotechnol. 2012, 4 (5), 492-510.

(24) Rapoport, N.; Gao, Z.; Kennedy, A. Multifunctional Nanoparticles for Combining Ultrasonic Tumor Imaging and Targeted Chemotherapy. JNCI J. Natl. Cancer Inst. 2007, 99 (14), 1095-1106.

(25) Chen, C. C.; Sheeran, P. S.; Wu, S. Y.; Olumolade, O. O.; Dayton, P. A.;

Konofagou, E. E. Targeted drug delivery with focused ultrasound-induced bloodbrain barrier opening using acoustically-activated nanodroplets. J. Controlled Release 2013, 172, 795-804.

(26) Zhang, X.; Hu, J.; Zhao, G.; Huang, N.; Tan, Y.; Pi, L.; Huang, Q.; Wang, F.; Wang, Z.; Wang, Z.; et al. PEGylated PLGA-Based Phase Shift Nanodroplets Combined with Focused Ultrasound for Blood Brain Barrier Opening in Rats. Oncotarget 2017, 8 (24), 38927-38936.

(27) Wu, S.-Y.; Fix, S. M.; Arena, C.; Chen, C. C.; Zheng, W.; Olumolade, O. O.; Papadopoulou, V.; Novell, A.; Dayton, P. A.; Konofagou, E. E. Focused Ultrasound-Facilitated Brain Drug Delivery Using Optimized Nanodroplets: Vaporization Efficiency Dictates Large Molecular Delivery. Phys. Med. Biol. 2018, 63 (3), 035002.

(28) Cheng, Y.; Dai, Q.; Morshed, R. A.; Fan, X.; Wegscheid, M. L.; Wainwright, D. A.; Han, Y.; Zhang, L.; Auffinger, B.; Tobias, A. L.; et al. Blood Brain Barrier Permeable Gold Nanoparticles: An Efficient Delivery Platform for Enhanced Malignant Glioma Therapy and Imaging. Small 2014, 10 (24), 5137-5150.

(29) Sykes, E. A.; Chen, J.; Zheng, G.; Chan, W. C. W. Investigating the Impact of Nanoparticle Size on Active and Passive Tumor Targeting Efficiency. ACS Nano 2014, 8 (6), 5696-5706.

(30) Cao, Y.; Chen, Y.; Yu, T.; Guo, Y.; Liu, F.; Yao, Y.; Li, P.; Wang, D.; Wang, Z.; Chen, Y.; et al. Drug Release from Phase-Changeable Nanodroplets Triggered by Low-Intensity Focused Ultrasound. Theranostics 2018, 8 (5), 1327-1339. 
(31) Park, Y.; Luce, A. C.; Whitaker, R. D.; Amin, B.; Cabodi, M.; Nap, R. J.; Szleifer, I.; Cleveland, R. O.; Nagy, J. O.; Wong, J. Y. Tunable Diacetylene Polymerized Shell Microbubbles as Ultrasound Contrast Agents. Langmuir 2012, 28 (8), 3766-3772.

(32) Chen, X.; Zhou, G.; Peng, X.; Yoon, J. Biosensors and Chemosensors Based on the Optical Responses of Polydiacetylenes. Chem. Soc. Rev. 2012, 41 (13), 4610-4630.

(33) Guo, C.; Liu, S.; Jiang, C.; Li, W.; Dai, Z.; Fritz, H.; Wu, X. A Promising Drug Controlled-Release System Based on Diacetylene/Phospholipid Polymerized Vesicles. Langmuir 2009, 25 (22), 13114-13119.

(34) Lee, H.-Y.; Tiwari, K. R.; Raghavan, S. R. Biopolymer Capsules Bearing Polydiacetylenic Vesicles as Colorimetric Sensors of PH and Temperature. Soft Matter 2011, 7 (7), 3273-3276.

(35) Chen, Y.-S.; Yoon, S. J.; Frey, W.; Dockery, M.; Emelianov, S. Dynamic Contrast-Enhanced Photoacoustic Imaging Using Photothermal StimuliResponsive Composite Nanomodulators. Nat. Commun. 2017, 8, 15782.

(36) Toumia, Y.; Cerroni, B.; Trochet, P.; Lacerenza, S.; Oddo, L.; Domenici, F.; Paradossi, G. Performances of a Pristine Graphene-Microbubble Hybrid Construct as Dual Imaging Contrast Agent and Assessment of Its Biodistribution by Photoacoustic Imaging. Part. Part. Syst. Charact. 2018, 35 (7), 1800066.

(37) Ishijima, A.; Minamihata, K.; Yamaguchi, S.; Yamahira, S.; Ichikawa, R.; Kobayashi, E.; Iijima, M.; Shibasaki, Y.; Azuma, T.; Nagamune, T.; et al. Selective Intracellular Vaporisation of Antibody-Conjugated Phase-Change Nano-Droplets in Vitro. Sci. Rep. 2017, 7, 44077.

(38) Ho, Y.-J.; Yeh, C.-K. Theranostic Performance of Acoustic Nanodroplet Vaporization-Generated Bubbles in Tumor Intertissue. Theranostics 2017, 7 (6), 1477-1488.

(39) Astafyeva, K.; Somaglino, L.; Desgranges, S.; Berti, R.; Patinote, C.; Langevin, D.; Lazeyras, F.; Salomir, R.; Polidori, A.; Contino-Pépin, C.; et al. Perfluorocarbon Nanodroplets Stabilized by Fluorinated Surfactants: Characterization and Potentiality as Theranostic Agents. J. Mater. Chem. B 2015, 3 (14), 2892-2907,

(40) Rapoport, N. Y.; Nam, K.-H.; Gao, Z.; Kennedy, A. Application of Ultrasound for Targeted Nanotherapy of Malignant Tumors. Acoust. Phys. 2009, 55 (4-5), 594-601.

(41) Fasolato, C.; Giantulli, S.; Silvestri, I.; Mazzarda, F.; Toumia, Y.; Ripanti, F.; Mura, F.; Luongo, F.; Costantini, F.; Bordi, F.; et al. Folate-Based Single Cell Screening Using Surface Enhanced Raman Microimaging. Nanoscale 2016, 8 (39), 17304-17313.

(42) Lange, H.; Rulli, F.; Crestini, C. Gel Permeation Chromatography in Determining Molecular Weights of Lignins: Critical Aspects Revisited for Improved Utility in the Development of Novel Materials. ACS Sustain. Chem. Eng. 2016, 4 (10), 5167-5180.

(43) Zabell, S. L.; Stigler, S. M.; Aldrich, J.; Edwards, A. W. F.; Seneta, E.; Diaconis, P.; Lehmann, E. On Student's 1908 Article "The Probable Error of a Mean" [with Comments, Rejoinder]. J. Am. Stat. Assoc. 2008, 103 (481), 1-20.

(44) Choi, H.; Bae, Y. M.; Yu, G. S.; Huh, K. M.; Choi, J. S. Synthesis of Poly(ethylene glycol)-Polydiacetylene Conjugates and Their Micellar and Chromic Characteristics. J. Nanosci. Nanotechnol. 2008, 8, 5104-5108, 
(45) Yarimaga, O.; Yoon, B.; Ham, D.-Y.; Lee, J.; Hara, M.; Choi, Y.-K.; Kim, J.-M. Electrophoretic Deposition of Amphiphilic Diacetylene Supramolecules: Polymerization, Selective Immobilization, Pattern Transfer and Sensor Applications. J. Mater. Chem. 2011, 21 (46), 18605-18612.

(46) Kim, Y.-H.; Subramanyam, E.; Im, J. H.; Huh, K. M.; Choi, H.; Choi, J. S.; Lee, Y.-K.; Park, S.-W. A New PEG-Lipid Conjugate Micelle for Encapsulation of CdSe/ZnS Quantum Dots. J. Nanosci. Nanotechnol. 2010, 10, 3275-3279.

(47) Araghi, H. Y.; Paige, M. F. The Effect of Perfluorotetradecanoic Acid on the Structure of Photopolymerized 10,12-Pentacosadiynoic Acid Films at the AirWater Interface. Can. J. Chem. 2013, 91 (11), 1130-1138.

(48) Vinod, T. P.; Chang, J.-H.; Kim, J.-K.; Rhee, S.-W. Self-Assembly and Photopolymerization of Diacetylene Molecules on Surface of Magnetite Nanoparticles. Bull. Korean Chem. Soc. 2008, 29 (4), 799-804.

(49) Wang, S.; Li, Y.; Shao, L.; Ramirez, J.; Wang, P. G.; Leblanc, R. M. Excess Free Energies of Interaction between 10,12-Pentacosadiynoic Acid (PDA) and Its Mannoside Derivative (MPDA). A Mixed-Monolayer Study. Langmuir 1997, 13 (6), 1677-1681. https://doi.org/10.1021/la9606411.

(50) Sasaki, D. Y.; Carpick, R. W.; Burns, A. R. High Molecular Orientation in Mono- and Trilayer Polydiacetylene Films Imaged by Atomic Force Microscopy. J. Colloid Interface Sci. 2000, 229 (2), 490-496. https://doi.org/10.1006/jcis.2000.7043.

(51) Sheeran, P. S.; Yoo, K.; Williams, R.; Yin, M.; Foster, F. S.; Burns, P. N. More Than Bubbles: Creating Phase-Shift Droplets from Commercially Available Ultrasound Contrast Agents. Ultrasound Med. Biol. 2017, 43 (2), 531-540.

(52) Mountford, P. A.; Borden, M. A. On the Thermodynamics and Kinetics of Superheated Fluorocarbon Phase-Change Agents. Adv. Colloid Interface Sci. 2016, 237, 15-27. https://doi.org/10.1016/j.cis.2016.08.007.

(53) Sheeran, P. S.; Luois, S. H.; Mullin, L. B.; Matsunaga, T. O.; Dayton, P. A. Design of Ultrasonically-Activatable Nanoparticles Using Low Boiling Point Perfluorocarbons. Biomaterials 2012, 33 (11), 3262-3269.

(54) Reznik, N.; Williams, R.; Burns, P. N. Investigation of Vaporized Submicron Perfluorocarbon Droplets as an Ultrasound Contrast Agent. Ultrasound Med. Biol. 2011, 37 (8), 1271-1279.

(55) Kripfgans, O. D.; Fowlkes, J. B.; Miller, D. L.; Eldevik, O. P.; Carson, P. L. Acoustic Droplet Vaporization for Therapeutic and Diagnostic Applications. Ultrasound Med. Biol. 2000, 26 (7), 1177-1189.

(56) D'Arrigo, J. S.; Imae, T. Physical Characteristics of Ultrastable Lipid-Coated Microbubbles. J. Colloid Interface Sci. 1992, 149 (2), 592-595.

(57) Fabiilli, M. L.; Haworth, K. J.; Fakhri, N. H.; Kripfgans, O. D.; Carson, P. L.; Fowlkes, J. B. The Role of Inertial Cavitation in Acoustic Droplet Vaporization. IEEE Trans. Ultrason. Ferroelectr. Freq. Control 2009, 56 (5), 1006-1017.

(58) Li, H.; Yang, Y.; Zhang, M.; Yin, L.; Tu, J.; Guo, X.; Zhang, D. Acoustic Characterization and Enhanced Ultrasound Imaging of Long-Circulating LipidCoated Microbubbles. J. Ultrasound Med. 2018, 37 (5), 1243-1256.

(59) Parrales, M. A.; Fernandez, J. M.; Perez-Saborid, M.; Kopechek, J. A.; Porter, T. M. Acoustic Characterization of Monodisperse Lipid-Coated Microbubbles: Relationship between Size and Shell Viscoelastic Properties. J. Acoust. Soc. Am. 2014, 136 (3), 1077-1084.

(60) Jingqi, W.; Lu, Z.; Jun, Z.; Yuhong, M.; Wei, Y.; Lifeng, R.; Chengbing, J.; Dobromir, D. D.; Hui, Z.; Kun, Z. Clinical Usefulness of the Microbubble 
Contrast Agent SonoVue in Enhancing the Effects of High-Intensity Focused Ultrasound for the Treatment of Adenomyosis. J. Ultrasound Med. 2018. https://doi.org/10.1002/jum.14638.

(61) Ferraioli, G.; Meloni, M. F. Contrast-Enhanced Ultrasonography of the Liver Using SonoVue. Ultrasonography 2018, 37 (1), 25-35.

(62) Harjanto, D.; Lee, J.; Kim, J.-M.; Jaworski, J. Controlling and Assessing the Surface Display of Cell-Binding Domains on Magnetite Conjugated Fluorescent Liposomes. Langmuir 2013, 29 (25), 7949-7956.

(63) Yun, J. S.; Yang, K. S.; Kim, D. H. Multifunctional Polydiacetylene-Graphene Nanohybrids for Biosensor Application. Journal of Nanoscience and Nanotechnology. 2011, 11, 5663-5669.

(64) Lee, J.; Jun, H.; Kim, J. Polydiacetylene-Liposome Microarrays for Selective and Sensitive Mercury(II) Detection. Adv. Mater. 2009, 21 (36), 3674-3677.

(65) Guo, C.; Liu, S.; Jiang, C.; Li, W.; Dai, Z.; Fritz, H.; Wu, X. A Promising Drug Controlled-Release System Based on Diacetylene/Phospholipid Polymerized Vesicles. Langmuir 2009, 25 (22), 13114-13119.

(66) Veneti, E.; Tu, R. S.; Auguste, D. T. RGD-Targeted Liposome Binding and Uptake on Breast Cancer Cells Is Dependent on Elastin Linker Secondary Structure. Bioconjug. Chem. 2016, 27 (8), 1813-1821.

(67) Meyer, A.; Auernheimer, J.; Kessler, A. M. and H. Targeting RGD Recognizing Integrins: Drug Development, Biomaterial Research, Tumor Imaging and Targeting. Curr. Pharm. Design 2006, 12, 2723-2747.

(68) Wu, P.-H.; Onodera, Y.; Ichikawa, Y.; Rankin, E. B.; Giaccia, A. J.; Watanabe, Y.; Qian, W.; Hashimoto, T.; Shirato, H.; Nam, J.-M. Targeting integrins with RGD-conjugated gold nanoparticles in radiotherapy decreases the invasive activity of breast cancer cells. Int. J. Nanomedicine 2017, 12, 5069-5085.

(69) Garanger, E.; Dumy, D. B. and P. Tumor Targeting with RGD Peptide Ligands-Design of New Molecular Conjugates for Imaging and Therapy of Cancers. Anti-Cancer Agents Med. Chem. 2007, 7, 552-558.

(70) Salvati, A.; Pitek, A. S.; Monopoli, M. P.; Prapainop, K.; Bombelli, F. B.; Hristov, D. R.; Kelly, P. M.; Åberg, C.; Mahon, E.; Dawson, K. A. TransferrinFunctionalized Nanoparticles Lose Their Targeting Capabilities When a Biomolecule Corona Adsorbs on the Surface. Nat. Nanotechnol. 2013, 8 (2), 137-143.

(71) Suk, J. S.; Xu, Q.; Kim, N.; Hanes, J.; Ensign, L. M. PEGylation as a Strategy for Improving Nanoparticle-Based Drug and Gene Delivery. Adv. Drug Deliv. Rev. 2016, 99, 28-51.

(72) Cheng, Y.; Dai, Q.; Morshed, R. A.; Fan, X.; Wegscheid, M. L.; Wainwright, D. A.; Han, Y.; Zhang, L.; Auffinger, B.; Tobias, A. L.; et al. Blood Brain Barrier Permeable Gold Nanoparticles: An Efficient Delivery Platform for Enhanced Malignant Glioma Therapy and Imaging. Small 2014, 10 (24), 5137-5150. 


\section{TABLE OF CONTENT}

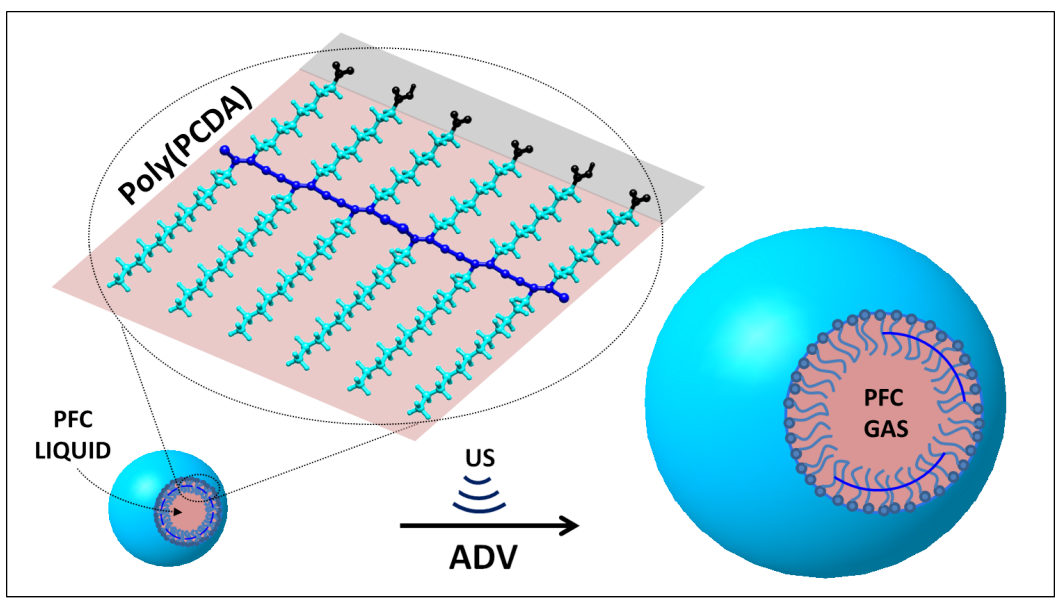

20

21 